\title{
Interplay of Monocytes and T Lymphocytes in COVID-19 Severity
}

Lindsey E. Padgett ${ }^{1,4}$, Huy Q. Dinh ${ }^{1,4}$, Serena J. Chee ${ }^{2,4}$, Claire E. Olingy ${ }^{1}$, Runpei Wu ${ }^{1}$, Daniel J. Araujo $^{1}$, Pandurangan Vijayanand ${ }^{1}$, Christian H. Ottensmeier ${ }^{2,3^{*}}$, and Catherine C. Hedrick ${ }^{1 *}$

${ }^{1}$ Center for Autoimmunity and Inflammation, La Jolla Institute for Immunology, La Jolla CA, 92037, USA.

${ }^{2}$ Faculty of Medicine, University of Southampton, Southampton, UK.

${ }^{3}$ Institute of Translational Medicine, Department of Molecular \& Clinical Cancer Medicine, University of Liverpool, Liverpool, UK.

${ }^{4}$ These authors jointly contributed to the work.

* Corresponding Authors:

Catherine C. Hedrick

La Jolla Institute for Allergy and Immunology

9420 Athena Circle,

La Jolla, CA 92037

Hedrick@1ji.org

Phone: 858-752-6500

and

Christian H. Ottensmeier

Institute of Translational Medicine

Department of Molecular \& Clinical Cancer Medicine

University of Liverpool

Liverpool, UK

cottensmeier@1ji.org 
bioRxiv preprint doi: https://doi.org/10.1101/2020.07.17.209304; this version posted July 18, 2020. The copyright holder for this preprint (which was not certified by peer review) is the author/funder, who has granted bioRxiv a license to display the preprint in perpetuity. It is made available under aCC-BY-NC-ND 4.0 International license.

One Sentence Summary: Use of mass cytometry on peripheral blood mononuclear cells from convalescent COVID-19 patients allows correlation of distinct monocyte and T lymphocyte subsets with clinical factors. 


\section{ABSTRACT}

The COVID-19 pandemic represents an ongoing global crisis that has already impacted over 13 million people. The responses of specific immune cell populations to the disease remain poorly defined, which hinders improvements in treatment and care management. Here, we utilized mass cytometry (CyTOF) to thoroughly phenotype peripheral myeloid cells and T lymphocytes from 30 convalescent patients with mild, moderate, and severe cases of COVID-19. We identified 10 clusters of monocytes and dendritic cells and 17 clusters of $\mathrm{T}$ cells. Examination of these clusters revealed that both $\mathrm{CD} 14^{+} \mathrm{CD} 16^{+}$ intermediate and $\mathrm{CD} 14{ }^{\mathrm{dim}} \mathrm{CD} 16^{+}$nonclassical monocytes, as well as $\mathrm{CD}^{+}$stem cell memory $\mathrm{T}\left(\mathrm{T}_{\mathrm{SCM}}\right)$ cells, correlated with COVID-19 severity, coagulation factor levels, and/or inflammatory indicators. We also identified two nonclassical monocyte subsets distinguished by expression of the sugar residue 6Sulfo LacNac (Slan). One of these subsets (Slan ${ }^{\text {lo }}$, nMo1) was depleted in moderately and severely ill patients, while the other (Slan $\left.{ }^{\text {hi }}, \mathrm{nMo} 2\right)$ increased with disease severity and was linked to $\mathrm{CD} 4^{+} \mathrm{T}$ effector memory $\left(\mathrm{T}_{\mathrm{EM}}\right)$ cell frequencies, coagulation factors, and inflammatory indicators. Intermediate monocytes tightly correlated with loss of naive $\mathrm{T}$ cells as well as an increased abundance of effector memory $\mathrm{T}$ cells expressing the exhaustion marker PD-1. Our data suggest that both intermediate and non-classical monocyte subsets shape the adaptive immune response to SARS-CoV-2. In summary, our study provides both broad and in-depth characterization of immune cell phenotypes in response to COVID-19 and suggests functional interactions between distinct cell types during the disease. 


\section{INTRODUCTION}

The coronavirus disease 2019 (COVID-19) pandemic, caused by SARS-Coronavirus-2 (SARSCoV-2), has currently affected more than 13 million people and claimed over 580,000 lives. Clinical presentation of COVID-19 varies, with some individuals exhibiting mild to severe symptoms while others remain asymptomatic. Severe cases are associated with acute respiratory distress syndrome, which requires hospitalization and mechanically-assisted ventilation (1). COVID-19 is also generally linked to an increased risk of cardiovascular events such as thrombosis (1-4), pulmonary embolism (3, 4), and stroke $(3,4)$, especially in critically ill patients. Comorbidities such as diabetes, hypertension, respiratory illness, and age (> 65 years) are common in severe cases $(1,5-9)$ and worsen prognosis $(5,8,10)$. Improved treatment of COVID-19 therefore requires a refined understanding of the heterogeneity in immune responses to SARS-CoV-2 infection and the cellular subsets that drive these processes.

Peripheral inflammation is linked to development of severe COVID-19 (11), and secondary immune mechanisms may regulate progression of the disease (12). Monocytes are myeloid cells that govern innate immune responses to viral infection and have traditionally been classified as $\mathrm{CD} 14^{+} \mathrm{CD} 16^{-}$ (classical), $\mathrm{CD} 14^{\mathrm{lo}} \mathrm{CD} 16^{+}$(nonclassical), and $\mathrm{CD} 14^{+} \mathrm{CD} 16^{+}$(intermediate). Expansion of CD14 ${ }^{+} \mathrm{HLA}-$ $\mathrm{DR}^{\mathrm{lo}}, \mathrm{CD} 14^{+} \mathrm{CD} 16^{+}$, and immunosuppressive, immature monocytes have been demonstrated in peripheral blood mononuclear cells (PBMCs) from severely ill COVID-19 patients $(11,13,14)$. Patients with mild forms of COVID-19 display an increased abundance of ${\text { CD } 14^{+}{ }^{+} \text {LA-DR }}^{\text {hi }}$ CD $11 c^{\text {hi }}$ inflammatory monocytes (14). T cells are lymphocytes that coordinate adaptive immune responses to viral insults and bifurcate into $\mathrm{CD}^{+}$(helper) and $\mathrm{CD}^{+}$(cytotoxic) subsets. Activation of peripheral $\mathrm{CD}^{+}$and $\mathrm{CD} 4^{+} \mathrm{T}$ cell subsets is heterogeneous amongst COVID-19 patients (15). Circulating $\mathrm{T}$ cell numbers are also broadly reduced in moderate to severe cases, with $\mathrm{CD}^{+} \mathrm{T}$ cells being particularly affected (15-17). Still, as there are conflicting reports on the expression of exhaustion markers on $\mathrm{T}$ cells during the course of the disease $(15,18-20)$, the events controlling this depletion are unknown. In-depth profiling of myeloid and $\mathrm{T}$ cell populations from COVID-19 patients could allow for interrogation of these processes, 
identification of other cellular subsets altered during illness, and correlation of cellular phenotypes with clinical parameters.

Herein, we utilize cytometry by time-of-flight (CyTOF) to assess myeloid and T cell heterogeneity in PBMCs from mildly to severely ill COVID-19 patients. We used these data to characterize the interplay between these two cell types in disease severity. Specifically, we identify unique subpopulations of monocytes and $\mathrm{T}$ cells and find that their relative abundances are differentially correlated to disease intensity and clinical factors. In summary, our work highlights perturbations in both monocytes and T lymphocytes as a response to COVID-19 and suggests interactions between these two immune cell types during the disease. 


\section{RESULTS}

\section{Global Changes in Immune Cell Composition and Responses in COVID-19}

PBMCs were obtained from the blood of 30 COVID-19 subjects during convalescence and analyzed using mass cytometry (Fig. 1A). Mildly ill patients $(n=8)$ were healthcare workers who tested positive for SARS-CoV-2 and became ill with COVID-19, but did not need hospitalization. The moderate $(n=13)$ and severe $(n=9)$ COVID-19 patient groups required hospitalization, with severely ill patients specifically requiring admittance to an intensive treatment unit (Fig. 1B). Patient demographics are detailed in Methods. Clinical data were obtained for moderately and severely ill patients while in hospital, and all patients in both groups presented with a clinically-defined inflammatory syndrome. We have limited clinical information on mildly ill COVID-19 individuals, as they were not hospitalized. Thus, the focus of our study was to determine what immune system perturbations distinguish moderate and severe cases of COVID-19.

Severely ill patients showed statistically significant increases in total white cell counts (Fig. 1C; $* p=0.013$ ), lactate dehydrogenase (LDH, Fig. 1D; ***p=0.0004), ferritin (Fig. 1E; *p=0.049), and CReactive Protein (CRP; $* * p=0.0086$; Fig. $1 \mathrm{~F}$ ) compared to moderately ill patients. Both hemoglobin (Fig. $1 \mathrm{G} ; * * p=0.0018$ ) and albumin (Fig. $1 \mathrm{H} ; * * p=0.0065)$ levels were lower in severe disease. Severely affected patients also had higher plasma levels of D-dimers (Fig. 1I; ***p=0.0001) and international normalized ratio (INR) calculated from prothrombin time (Fig. $1 \mathrm{~J} ; * * * p=0.0002$ ). Platelet counts were also increased in severe disease (fig. S1A). While these latter data on platelet counts did not reach statistical significance, like D-dimers and INR, they are consistent with reports of increased coagulation events in COVID-19 patients (9). Oxygen saturation levels were also significantly reduced in severely ill patients (fig. S1B; **p=0.003). Neutrophil:lymphocyte and neutrophil:monocyte ratios did not change, even though there was a trend towards increased neutrophil counts in severely ill patients (fig. S1C-D). Similarly, monocyte:lymphocyte ratios remained unchanged (fig. S1E). Comorbidities in hospitalized 
COVID-19 patients were primarily cardiovascular in scope, and included prior history of myocardial infarction and hypertension (see Methods).

Using a 41-marker CyTOF panel focused on identifying monocyte and T lymphocyte changes in COVID-19 (fig. S1F), we first assessed global immune cell changes in patients stratified by illness severity. We then utilized FlowSOM, an unbiased clustering method (21), along with meta-clustering and consensus clustering (see Methods) to identify 9 major immune cell types projected onto a Uniform Manifold Approximation and Projection plot (UMAP) plot (22), via assessment of markers for conventional $\mathrm{T}$ and monocyte cell types (Fig. 1K-O; fig. S2). UMAPs of the expression levels of all markers used to identify each $\mathrm{CD} 45^{+}$immune cell subset can be found in Supplementary Figure 2. Frequencies of all major immune cell types identified within the $\mathrm{CD}^{+} 5^{+}$gate for mildly (green), moderately (blue), and severely (red) ill COVID-19 patients are shown (fig. S1G,H). Specifically, we identified $\mathrm{CD}^{+}, \mathrm{CD}^{+}$, as well as double positive/negative (DN/DP), and $\mathrm{CD}^{+} 6^{+}$cells in the $\mathrm{T}$ cell compartment (fig. S1G; Fig. 1K,L; fig. S2). Other major cell types were dendritic cells (DC), natural killer (NK) cells, CD123 ${ }^{+}$cells (likely plasmacytoid dendritic cells (pDCs)) and monocytes (fig. S1H) using marker expression combinations of CD14 (Fig. 1M), CD16 (Fig. 1N), HLA-DR, CD1c, and CD123 (fig. S2). As our study was focused on monocyte/DC and T cells, markers for eosinophils, neutrophils, and B cells present in PBMC were placed in the same channel for exclusion (CD19/Siglec8/CD66b). We observed a slight reduction in $\mathrm{CD}^{+}$and $\mathrm{CD}^{+} \mathrm{T}$ cell frequencies in severe COVID patients, which is consistent with previous reports $(15,16,23-26)$ (fig. $\mathrm{S} 1 \mathrm{G})$. We also found a significant increase in total monocyte frequencies stratified by disease severity, and a similar trend for $\mathrm{CD}_{1} \mathrm{c}^{+} \mathrm{CD} 11 \mathrm{c}^{\text {hi }} \mathrm{DCs}$ (fig. $\mathrm{S} 1 \mathrm{H})$.

\section{Classical Monocyte Heterogeneity in COVID-19}

Heterogeneous myeloid cell phenotypes have been observed in COVID-19 patients (13), but how these phenotypes are dictated by SARS-CoV-2 illness is unknown. We designed a CyTOF panel to examine monocyte and DC heterogeneity in-depth, as we have done for our work in profiling immune 
cells during atherosclerosis (27). All monocytes and DCs identified in Figure 1C were re-clustered and projected onto a new UMAP to obtain a detailed myeloid heterogeneity landscape (Fig. 2A; fig. S3). Using FlowSOM clustering and meta-clustering to merge subsets of these myeloid cells, we identified 10 clusters containing monocytes and DCs in our COVID-19 cohort. These included 1 DC cluster, four $\mathrm{CD}_{14}{ }^{+}$classical monocyte $(\mathrm{cMo})$ clusters, $1 \mathrm{CD}^{+} 4^{+} \mathrm{CD} 16^{+}$intermediate monocyte (iMo) cluster, and 2 $\mathrm{CD} 14^{10} \mathrm{CD} 16^{+}$nonclassical monocyte (nMo) clusters (Fig. 2A). We also observed 2 new monocyte subsets present in blood of patients with COVID-19: $\mathrm{CD}_{14}^{+} \mathrm{CD} 16^{+} \mathrm{CD} 56^{+}\left(\mathrm{CD} 56^{+}\right)$monocytes and $\mathrm{CD} 4^{+} \mathrm{CD}^{+}\left(\mathrm{CD}^{+}\right)$monocytes (Fig. 2A/C). $\mathrm{CD}^{+} 6^{+}$monocytes possess cytotoxic NK-like characteristics and have been identified in mice (28). A related $\mathrm{CD} 14^{+} \mathrm{CD} 56^{+}$subset that lacks $\mathrm{CD} 16$ and is highly phagocytic has been reported in humans (29). $\mathrm{CD}^{+}$monocytes were also identified, and we confirmed that these were not doublets or cell aggregates (data not shown). Expression of CyTOF markers present on each myeloid cell cluster is shown in a heatmap in Figure 2B. We also observed that enrichment of each cluster varied between patients; however, all patients possessed all clusters (Fig. 2C). The classical monocyte cluster cMo1 increased in both moderate and severe cases compared to mild cases (Fig. 2D). Additionally, the classical monocyte cluster cMo3 trended towards an increased abundance in both moderate and severe, compared to mild disease (Fig. 2D). Classical monocytes may contribute to the inflammation associated with COVID-19 severity, as they broadly upregulate activation markers during SARS-CoV-2 infection (14). In comparing markers defining the classical monocyte subsets (Fig. 2B and fig. S3), we observed that cMo1 and cMo2 have high CD33 expression. cMo1 also express CD45RA and CD163. cMo2 express CD9, HLA-DR, CD93, and CD36. cMo3 have moderate expression of CD33, CD36, and much lower expression of HLA-DR than do cMo1 and cMo2. Although we and others (30) observe an increase in classical monocytes amongst COVID-19 patients, the cMo1, cMo3 subsets specifically increased, whereas cMo2 decreased, with disease severity (Fig. 2D). cMo3 was the largest classical monocyte cluster in terms of cell frequency, and cMo4 was the smallest (Fig. 2A/D). cMo4 was abundant in several moderately and severely affected patients and was characterized by low expression of 
the siglec CD33, which is variably expressed in human PBMCs (31). CD56 ${ }^{+}$monocytes and $\mathrm{CD}^{+}$ monocytes also exhibited slight reductions with disease severity (Fig. 2D).

\section{Intermediate and Nonclassical Monocytes are Greatly Impacted by COVID-19}

The most robust changes observed for myeloid cells in our cohort were those impacting the intermediate and nonclassical monocyte subsets. Intermediate monocytes (iMo) are $\mathrm{CD}_{14}^{+} \mathrm{CD} 16^{+}$and elevated in atherosclerosis (32), linked to microinflammation in the vasculature in Type 2 diabetes (33), and associated with impaired vascular function in kidney disease (34). iMo are believed to represent a transitional monocyte stage through which classical $\mathrm{CD}_{1} 4^{+}$monocytes convert into nonclassical CD14 ${ }^{\mathrm{dim}} \mathrm{CD} 16^{+}$monocytes (35). Upon activation, classical monocytes are biased towards loss and gain of $\mathrm{CD}_{14}{ }^{+}$and $\mathrm{CD}_{16}{ }^{+}$expression, respectively (36). iMo cells express genes related to antigen presentation and participate in activation of the adaptive immune response, inflammation, and angiogenesis (37). In our COVID-19 cohort, the iMo cluster positively correlated with disease severity $(\mathrm{p}<0.05)$, with a 2.5fold increase in iMo frequencies in mildly compared to severely ill individuals (Fig. 2D). An increase in pro-inflammatory iMo that expressed both IL-6 and GM-CSF was also reported in hospitalized COVID19 patients in Hefei, China (38).

In contrast, $\mathrm{CD} 14{ }^{\mathrm{dim}} \mathrm{CD} 16^{+}$nonclassical monocytes (nMo) play critical roles in maintaining vascular homeostasis (39) and guard against viral pathogens (40). Shulte-Schrepping et al. (14) recently reported depletion of all nonclassical monocytes in COVID-19. However, we know from our prior studies in human cardiovascular disease that nonclassical monocytes consist of distinct subsets (27). Here, in our COVID-19 cohort, nonclassical monocytes clustered into two separate populations (Fig. 2A). One population (nMo2) was positive for 6-sulfo-Lac-Nac (Slan), a glycosylated form of P-selectin Glycoprotein-1 (PSGL-1) (Fig. 2B). Slan ${ }^{+}$nonclassical monocytes (called nMo2 here) have been identified in cancer (41), atherosclerosis (27), and multiple sclerosis (42), and were formerly designated as Slan-DCs (43). nMo2 produce IL-12 (44), IFN $\gamma$ (45), activate both antigen-specific CD4 ${ }^{+}$and cytotoxic $\mathrm{CD}^{+} \mathrm{T}$ cells $(46,47)$, enable tumor phagocytosis and killing $(41,48)$, and enhance NK cell 
activation $(48,49)$. Thus, $\mathrm{Slan}^{+}$nonclassical nMo2 monocytes regulate vascular homeostasis and drive immune responses. Here, we found that $\operatorname{Slan}^{+} \mathrm{CD} 16^{+}$nonclassical monocytes (nMo2) were nearly absent in both moderate and severe COVID-19 patients (Fig. 2C, light pink bars; Fig. 2D; $p=0.003$ and $p=0.01$, Wilcox rank-sum test). Loss of $\mathrm{nMo} 2$ could thus promote progression to moderate and severe COVID-19 illness.

The other nonclassical monocyte subset, nMo1, was increased 4-fold in severely ill patients compared to moderately ill patients; still, this population was significantly lower in the moderate compared to the mild disease group (Fig. 2D). The cells within nMo1 lowly express Slan, but a previous study from our laboratory demonstrated that they do express CD40, CD63, CD93, and CD36 (27). The nMo1 cluster in moderately affected patients also exhibited higher expression of HLA-DR, CD45RA, PDL1, and PD-L2 than that of mild or severe disease groups (Fig. 2B; fig. S3). CD45RA ${ }^{+}$nonclassical monocytes have been associated with increased low density lipoprotein (LDL)-cholesterol levels (29). Interestingly, CD45RA is also a marker of myeloid progenitor cells, often found present in blood in acute myeloid leukemia patients (50), further supporting dysregulation of the homeostatic myeloid response by SARS-CoV-2 infection. The presence of PD-L1 and PD-L2 on the nMo1 cluster in moderately ill patients (compared to patients with mild or severe disease) suggests that these cells are unable to mount an effective immune response and may contribute to T cell exhaustion. Expression of PD-L1 and PD-L2 on nMo1 is lower in severe patients, yet the mechanism for this reduction of PD-L1 and PD-L2 in severely ill patients is unknown. Finally, as we included only the DC markers CD11c, CD123, CD1c, and CD141 in our CyTOF panel, we only observed 1 DC subset (Fig. 2A). This DC subset was decreased in moderate and severe patients compared to mild disease (Fig. 2C) as expected and observed in other studies (15, 18).

\section{Monocyte Phenotypes Associated with Clinical Features of COVID-19}

We next asked if any monocyte or DC clusters were significantly associated with the clinical features of COVID-19 patients. We correlated myeloid cell frequencies with their matched inflammatory and coagulation parameters (shown in Fig. 1), as well as with COVID-19 severity, using Spearman rank 
correlations. DCs were negatively correlated with CRP levels $(\mathrm{r}=-0.62, p=0.003)$ and positively correlated with platelet counts ( $\mathrm{r}=0.45, p=0.036)$ upon patient arrival to hospital (Fig. $2 \mathrm{E})$. Of the classical monocyte clusters, cMo1 was associated with albumin levels, but this did not reach statistical significance. Thus, classical monocytes, although mostly increased in disease severity, were not stringently linked to any clinical parameter measured in our patients. In contrast, iMo were tightly and positively associated with disease severity (Fig. 2D), but were not significantly linked with any specific clinical parameters. There was a minor negative association with oxygen saturation supporting association with disease severity, but this was not significant ( $\mathrm{r}=-0.4, p=0.06$; data not shown).

The most striking findings arose from our analyses related to nonclassical monocyte phenotypes. nMo1 frequencies were higher in severely ill patients (Fig. 2D), positively correlated to the inflammatory marker LDH ( $\mathrm{r}=0.45, p=0.04)$ (Fig. $2 \mathrm{~F})$, negatively correlated to levels of the coagulation indicator INR $(\mathrm{r}=0.54, p=0.009)$ (Fig. 2F), and positively correlated to D-dimers ( $\mathrm{r}=0.56, p=0.008)$ (Fig. 2F) in these individuals. There was also a trend towards positive correlation of nMo1 with inflammatory CRP levels $(\mathrm{r}=0.37, p=0.08$, data not shown). These data indicate that $\mathrm{nMo1}$ levels are tightly associated with clinical parameters of coagulation and inflammation. Unfortunately, numbers of nMo2 were too low to power the assessment of associations of this cell type with disease severity.

\section{T Cell Heterogeneity in COVID-19 Patients}

We next examined perturbations in the $\mathrm{T}$ cell compartment with disease severity within our cohort. $\mathrm{CD}^{+} \mathrm{T}$ cells and $\mathrm{CD} 4^{+} \mathrm{T}$ cells were re-clustered and projected onto a UMAP to obtain a detailed look at $\mathrm{T}$ cell heterogeneity (Fig. 3A). Using FlowSOM clustering and meta-clustering for merging subsets, we identified 17 clusters of T cells in PBMCs from our COVID-19 cohort. Specifically, we identified $7 \mathrm{CD}^{+} \mathrm{T}$ cell subsets, $7 \mathrm{CD}^{+} \mathrm{T}$ cell subsets, a subset of $\mathrm{CD}^{+} \mathrm{T}$ cells that were negative for CD4 and CD8 (double-negative $(\mathrm{DN}), \mathrm{CD}^{+} \mathrm{CD} 8^{+} \mathrm{T}$ cells (double-positive, $\mathrm{DP}$ ), and $\mathrm{CD}^{+} \mathrm{CD}^{+} 6^{+} \mathrm{T}$ cells (Fig. 3A). Within the CD4 ${ }^{+} \mathrm{T}$ cell compartment, 7 major cell clusters emerged on the basis of CD45RA, CD45RO, CCR7, CXCR3, CD11a, CD73, CD95, and PD-1 (Fig. 3B; fig. S4). In particular, we identified 
a $\mathrm{CD}^{+} \mathrm{T}$ naive $\left(\mathrm{T}_{\mathrm{N}}\right)\left(\mathrm{CD} 45 \mathrm{RA}^{+} \mathrm{CCR} 7^{+} \mathrm{CD} 45 \mathrm{RO}{ }^{\text {lo }} \mathrm{CD} 95^{\text {lo }}\right)$ subset, a stem cell memory $\mathrm{T}\left(\mathrm{T}_{\mathrm{SCM}}-\right.$

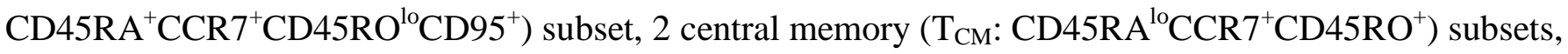
2 effector memory $\left(\mathrm{T}_{\mathrm{EM}}\right.$ : $\left.\mathrm{CD} 45 \mathrm{RA}{ }^{\mathrm{lo}} \mathrm{CCR} 7^{\mathrm{lo}} \mathrm{CD} 45 \mathrm{RO}^{+}\right)$subsets, and a terminally differentiated effector $\left(\mathrm{T}_{\text {EMRA }}\right.$ : $\mathrm{CD}^{4} 5 \mathrm{RA}^{+} \mathrm{CCR}{ }^{-} \mathrm{CD} 45 \mathrm{RO}^{\mathrm{lo}}$ ) subset. The $2 \mathrm{~T}_{\mathrm{CM}}$ subsets were distinguished by low and high expression of the inflammatory chemokine receptor CXCR3. As the receptor for CXCL9, CXCL10, and CXCL11, CXCR3 is highly expressed within effector and memory subsets but largely absent on CD4 ${ }^{+} \mathrm{T}_{\mathrm{N}}$ cells (51). CD4 ${ }^{+} \mathrm{T}$ helper 1 (Th1) cells have robust expression of CXCR3 (Fig. 3B) (52). Thus, we surmise that the $\mathrm{CXCR}^{+} \mathrm{T}_{\mathrm{CM}}$ subset $\left(\mathrm{T}_{\mathrm{CM}} 1\right)$ is Th1-like. The 2 remaining $\mathrm{CD} 4^{+} \mathrm{T}_{\mathrm{EM}}$ subsets were distinguished by high and low PD-1 expression (Fig. 3B). PD-1 is associated with T cell exhaustion, but also upregulated on $\mathrm{T}$ cells that have recently encountered cognate antigen.

Within the $\mathrm{CD}^{+} \mathrm{T}$ cell compartment, we identified $2 \mathrm{CD}^{+} \mathrm{T}_{\mathrm{N}}$ subsets, a $\mathrm{CD} 8^{+} \mathrm{T}_{\mathrm{SCM}}$ subset, 1 $\mathrm{CD}^{+} \mathrm{T}_{\mathrm{CM}^{-}}$like subset, $2 \mathrm{CD}^{+} \mathrm{T}_{\mathrm{EM}}$ subsets, and $1 \mathrm{CD}^{+} \mathrm{T}_{\mathrm{EMRA}}$ subset (Fig. 3B; fig. S4). The $2 \mathrm{CD}^{+} \mathrm{T}_{\mathrm{N}}$ subsets were distinguished by the pro-inflammatory chemokine receptor CXCR3. $\mathrm{CD}^{+} \mathrm{T}_{\mathrm{N}}$ cells expressing CXCR3 possess increased effector potential compared to $\mathrm{T}_{\mathrm{N}}$ cells (53). Importantly, these cells are distinct from $\mathrm{CD}^{+} \mathrm{T}_{\mathrm{SCM}}$ cells, as they lack CD95 expression. The $\mathrm{CD} 8^{+} \mathrm{T}_{\mathrm{SCM}}$ subset expressed the $\mathrm{T}_{\mathrm{N}}$ markers CD45RA, CCR7, and CD95. CXCR3 was also noted within this $\mathrm{CD}^{+} \mathrm{T}_{\mathrm{SCM}}$ population and is a known marker of these cells $(54,55)$. The $\mathrm{CD}^{+} \mathrm{T}_{\mathrm{CM}}$-like subset was CD45RA ${ }^{\text {lo }}$ and also expressed CCR7; however, expression of CD45RO was quite dim within the $\mathrm{CD}^{+} \mathrm{T}$ cell compartment overall (fig. $\mathrm{S} 3$ ). This $\mathrm{CD}^{+} \mathrm{T}_{\mathrm{CM}^{-}}$like cell was noted by high expression of the ecto-5' nucleotidase $\mathrm{CD} 73$ and CXCR3. The $\mathrm{CD}^{+} \mathrm{T}_{\mathrm{EM}}$ subset 2 was noted by high expression of the $\mathrm{T}$ cell exhaustion marker PD-1 and HLA-DR, whereas these markers were lowly expressed within $\mathrm{CD}^{+} \mathrm{T}_{\mathrm{EM}} 1$. Similar to the $\mathrm{CD}^{+} \mathrm{T}_{\mathrm{CM}^{-}}$like subset, expression of CD45RO was dim with $\mathrm{CD}^{+}$TEM 1, but CD45RA and CCR7 were not expressed. Analysis of subset frequencies revealed that these 17 subsets were expressed within all mildly, moderately, and severely ill individuals (Fig. 3C).

\section{Changes in T Cell Subsets with COVID-19 Severity}


When quantifying $\mathrm{CD}^{+} \mathrm{T}$ cell subset frequencies in all patient groups, we observed significantly attenuated (3-fold) frequencies of the $\mathrm{CD}^{+} \mathrm{T}_{\mathrm{CM}} 1$ subset associated with high CXCR3 expression within mildly compared to moderately ill individuals (Fig. 3D). These $\mathrm{T}_{\mathrm{CM}}$ cells, noted by high CXCR3 expression may embody a Th1-like subset. In anti-viral responses, Th1-polarized CD4 T cells propagate anti-viral $\mathrm{CD}^{+} \mathrm{T}$ cell responses. Our study indicates that this Th1 subset is lost in moderate disease. We also noted a significant (9-fold) enrichment in the $\mathrm{CD}^{+} \mathrm{T}_{\mathrm{EMRA}}$ cell subset within moderately compared to mildly ill individuals (Fig. 3D). A recent study identified a significant increase in $C D 4^{+} \mathrm{T}_{\mathrm{EMRA}}$ frequencies in COVID-19 individuals compared to age-matched healthy controls; however, COVID-19 subjects in this study were not stratified by disease severity (26). Thus, moderately ill COVID-19 individuals display a loss of $\mathrm{CD}^{+} \mathrm{CXCR}^{\text {hi }} \mathrm{T}_{\mathrm{CM}}$ cells and increased $\mathrm{T}_{\mathrm{EMRA}}$ cells.

Examination of the $\mathrm{CD}^{+} \mathrm{T}$ cell compartment revealed 1.75 -fold attenuations in the CXCR3 ${ }^{\text {lo }}$ $\mathrm{CD}^{+} \mathrm{T}_{\mathrm{N}}$ cell subset 1 in mildly compared to moderately ill and mildly compared to severely ill individuals (Fig. 3D). Moreover, the more effector-like CXCR $3^{+} \mathrm{CD} 8^{+} \mathrm{T}_{\mathrm{N}}$ subset 2 was decreased (6.6 and 8.8-fold, respectively) within mildly compared to moderately and severely affected individuals, respectively (Fig. 3D). The $\mathrm{CD} 8^{+} \mathrm{T}_{\mathrm{CM}^{-}}$like subset was significantly decreased by 1.7 - and 1.8 -fold within mildly compared to moderately and mildly compared to severely affected individuals, respectively (Fig. 3D). Moreover, the $\mathrm{CD}^{+} \mathrm{T}_{\mathrm{EM}} 1$ subset was increased within mildly compared to moderately ill individuals. Thus, severely ill COVID-19 individuals displayed enriched $\mathrm{CD}^{+} \mathrm{T}_{\mathrm{EM}} / \mathrm{T}_{\mathrm{EMRA}}$ populations and diminished $\mathrm{T}_{\mathrm{N}}$ subsets.

Analysis of our cohort (see Methods) confirmed that the mean age of non-hospitalized individuals with mild disease was significantly lower than that of the moderately-severely ill patients $(* * * p=0.0004$ (mild vs. moderate), ${ }^{*} p=0.0161$ (mild vs. severe), one-way ANOVA). As age is linked to $\mathrm{T}_{\mathrm{N}}$ and $\mathrm{T}$ memory cell frequencies $(56,57)$, we decided to focus this study only on changes observed in the hospitalized patients (moderately + severely ill individuals), who were similar in age. We observed a 2fold reduction in circulating $\mathrm{CD}^{+} \mathrm{T}_{\mathrm{SCM}}$ cells in moderate compared to severe COVID-19 disease (Fig. 
3D). Human $\mathrm{T}_{\mathrm{SCM}}$ cells, the least differentiated memory subset, are phenotyped as $\mathrm{T}_{\mathrm{N}}$ cells that express CD95. $\mathrm{T}_{\mathrm{SCM}}$ cells are highly proliferative, self-renewing, cytokine-producing, and endowed with the capacity to differentiate into $\mathrm{T}_{\mathrm{CM}}$ and $\mathrm{T}_{\mathrm{EM}}$ subsets $(58,59)$. Importantly, within the $\mathrm{T}$ cell compartment, this $\mathrm{CD}^{+} \mathrm{T}_{\mathrm{SCM}}$ cell cluster was the sole differentially-expressed subset between moderate and severe disease (Fig. 3D). Interestingly, while not statistically significant, $\mathrm{CD}^{+} \mathrm{T}_{\mathrm{SCM}}$ cell frequencies were also similarly reduced in moderately versus severely ill COVID-19 individuals (Fig. 3D).

\section{T Cell Phenotypes Associated with Clinical Parameters of COVID-19}

When correlated with clinical parameters, we observed a strong negative association of CD4 ${ }^{+}$ $\mathrm{T}_{\mathrm{SCM}}$ frequencies from mildly and moderately affected individuals with international normalized ratio (INR) calculated from prothrombin time $(\mathrm{r}=-0.43, p=0.0488)$, which was significantly reduced in severe disease (Fig. 1J; Fig. 3E). Conversely, $C D 4^{+} \mathrm{T}_{\mathrm{CM}} 2\left(\mathrm{CXCR} 3^{\mathrm{lo}}\right)$ frequencies were positively correlated with the inflammatory parameters lactate dehydrogenase $(\mathrm{LDH} ; \mathrm{r}=0.45, p=0.05)$ and ferritin $(\mathrm{r}=0.56$, $p=0.01)($ Fig $3 \mathrm{E}) . \mathrm{CD}^{+} \mathrm{T}_{\mathrm{EMRA}}$ frequencies were positively correlated with hemoglobin $(\mathrm{r}=0.45, p=0.04)$ (Fig. S5A). Examination of the $\mathrm{CD}^{+} \mathrm{T}$ cell compartment revealed that $\mathrm{CD}^{+} \mathrm{T}_{\mathrm{SCM}}$ cell frequencies were inversely correlated with INR ( $\mathrm{r}=-0.53, p=0.0016$; Fig. S5B), while $\mathrm{CD}^{+} \mathrm{T}_{\mathrm{EMRA}}$ frequencies were positively correlated with hemoglobin ( $\mathrm{r}=0.46, p=0.03$ ) (Fig. S5C) and albumin ( $\mathrm{r}=0.44, p=0.0382$; Fig. S5D).

\section{Interplay of Monocytes and T Cells in COVID-19 Severity}

We next asked if the frequencies of monocyte subsets were associated with frequencies of specific $\mathrm{T}$ cells and if these correlations were linked to disease severity. While classical monocytes were not strongly linked with specific clinical measurements in our cohort, we did find that cMo3 frequencies (only) showed statistically significant associations with $\mathrm{T}$ cell subsets. In particular, we observed a negative association of $\mathrm{cMo} 3$ with $\mathrm{CD}^{+} \mathrm{T}_{\mathrm{N}}$ cells $(\mathrm{r}=-0.51, p=0.0048$; Fig. 4A). The cMo3 cluster was also significantly associated with the following $\mathrm{T}$ cell subsets that were changed with COVID-19 severity or that correlated with inflammatory indicators: $\mathrm{CD}^{+} \mathrm{T}_{\mathrm{N}} 1$ cells, $\mathrm{CD} 4^{+} \mathrm{T}_{\mathrm{SCM}}$, as well as, $\mathrm{CD}^{+} \mathrm{T}_{\mathrm{CM}} 2$, 
$\mathrm{CD}^{+} \mathrm{T}_{\mathrm{EM}} 2$, and $\mathrm{CD} 8^{+} \mathrm{T}_{\mathrm{EM}} 2$. Specifically, the cMo3 cluster was negatively associated with $\mathrm{CD} 8^{+} \mathrm{T}_{\mathrm{N}} 1$ $\left(\mathrm{CXCR} 3^{\text {lo }}\right.$ ) and $\mathrm{CD}^{+} \mathrm{T}_{\mathrm{SCM}}$ cells (Fig. 4A). In contrast to negative associations with $\mathrm{T}_{\mathrm{N}}$ and $\mathrm{T}_{\mathrm{SCM}}, \mathrm{cMo} 3$ was positively associated with $\mathrm{CD}^{+} \mathrm{T}_{\mathrm{CM}} 2(\mathrm{r}=0.63, p=3 \mathrm{e}-4), \mathrm{CD}^{+} \mathrm{T}_{\mathrm{EM}} 2(\mathrm{r}=0.41 . p=0.0268)$, and $\mathrm{CD} 8^{+}$ $\mathrm{T}_{\mathrm{EM}} 2$ (r=0.46, p=0.011) (Fig. 4A). $\mathrm{CD}^{+} \mathrm{T}_{\mathrm{EM}} 2$ and $\mathrm{CD}^{+} \mathrm{T}_{\mathrm{EM}} 2$ may represent "exhausted" $\mathrm{T}$ cells, as these subsets were noted by PD-1 expression (Fig. 3B). A recent study found that stimulation of COVID19 PBMCs elicited a profound $\mathrm{CD}^{+} \mathrm{T}_{\mathrm{CM}}$ and a $\mathrm{CD} 8^{+} \mathrm{T}_{\mathrm{EM}} / \mathrm{T}_{\mathrm{EMRA}}$ response (60). Thus, cMo3 may contribute to immune dysregulation in response to COVID-19.

Additionally, we found that intermediate monocytes (iMo) showed the strongest correlation with T cell subsets that delineated disease severity (Fig. 4B). Specifically, iMo was negatively correlated with both $\mathrm{CD}^{+} \mathrm{T}_{\mathrm{N}}(\mathrm{r}=-0.51, p=0.0044)$ and $\mathrm{CXCR} 3^{\text {lo }} \mathrm{CD}^{+} \mathrm{T}_{\mathrm{N}}(\mathrm{r}=-0.42, p=0.0204)$ subsets, and positively correlated with corresponding effector $\mathrm{CD}^{+} \mathrm{T}_{\mathrm{EMRA}}(\mathrm{r}=0.4, p=0.0279)$, and the PD-1-expressing $\mathrm{CD}^{+} \mathrm{T}_{\mathrm{EM}}$ $2(\mathrm{r}=0.54, p=0.0026)$, and $\mathrm{CD}^{+} \mathrm{T}_{\mathrm{EM}} 2$ subsets $(\mathrm{r}=0.58, p=0.001)$ (Fig. 4B). Cells in the iMo cluster express high levels of HLA-DR, and relatively high expression of CD9. CD9 is a tetraspanin present in plasma membrane microdomains, including lipid rafts (61). CD9 in monocytes and DC regulates HLADR trafficking, thereby influencing T cell activation (61). Thus, the high expression of HLA-DR in iMo may drive antigen presentation, and ultimately contribute to T cell exhaustion in these patients with severe illness. As cells within iMo are considered a transitional state in monocyte development, and because we found that iMo frequencies are significantly associated with COVID-19 disease severity (Fig. 2D), the correlation of iMo with these $\mathrm{T}$ cell subsets indicates potential likely functional crosstalk between fluid monocyte states and T cells during different stages of illness severity.

The nMo1 cluster was positively correlated with $\mathrm{CD}^{+} \mathrm{T}_{\mathrm{EM}} 1$ cells $(\mathrm{r}=0.37, p=0.0048)$ (Fig. 4C). These effector memory $\mathrm{CD}^{+} \mathrm{T}$ cells displayed high expression of CD73 (5'-ectonucleotidase), an ectoenzyme that catalyzes the conversion of 5' AMP to immunosuppressive adenosine (62). CD73 is a marker of $\mathrm{T}$ regulatory (Treg) cells; $\mathrm{CD} 25^{\text {high }} \mathrm{CD} 127^{\mathrm{lo}} \mathrm{CD} 4^{+} \mathrm{T}$ cells that express the forkhead box transcription factor Foxp3 and the ectoenzyme CD39 (63). Thus, this CD4 ${ }^{+} \mathrm{T}_{\mathrm{EM}} 1$ subset is likely a Treg 
subset. However, as we didn't include the markers CD25, CD127, Foxp3, or CD39 in our CyTOF panel, we cannot fully define this subset. $\mathrm{CD}^{+} \mathrm{T}_{\mathrm{EM}} 1$ cells displayed trending reductions in frequency within mild compared to moderate and severe disease. In another study, Treg cells were attenuated in individuals with severe compared to mild COVID-19 (64). Similarly, our collaborators have found that antigenspecific SARS-COV2 reactive Treg cells are severely attenuated within hospitalized compared to nonhospitalized individuals (65). While lung tumor-infiltrating non-classical monocytes secrete chemokines that recruit $\mathrm{T}$ cells (48), the interplay between non-classical monocytes and Tregs is not well described. Further study is needed to determine the interplay between non-classical monocytes and Tregs during COVID-19.

Unfortunately, we were unable to perform correlations with either $\mathrm{nMo} 2$ or $\mathrm{cMo} 4$, due to their paucity in moderately and severely affected patients. In lieu of this method, we performed unsupervised clustering of PBMCs from all individuals by analyzing 487 combinations (10 myeloid subsets +24 markers, $17 \mathrm{~T}$ cell subsets +14 markers). Shown in Fig. S6 are the top differentially-expressed combinations of features across disease severity from this analysis. We found that CD141 expression in nMo2 (nMo2-CD14) and CD93 expression in both nMo1 and nMo2 (nMo2-CD93 and nMo1-CD93) best distinguished mildly ill patients (green bars) from the hospitalized patients (blue and red bars) (Fig. S6). Thus, the primary changes in the myeloid compartment that associate with COVID-19 severity lie in the nonclassical monocyte subsets. In addition, we observed a number of features that were dominantly expressed in moderately ill patients, such as PD1 expression in naive $\mathrm{CD}^{+}\left(\mathrm{CD}^{+} \mathrm{T}_{\mathrm{N}} 2-\mathrm{PD} 1\right)$ and $\mathrm{CXCR} 3$ in $\mathrm{CD}^{+} \mathrm{T}_{\mathrm{CM}}\left(\mathrm{CD}^{+} \mathrm{T}_{\mathrm{CM}}\right.$ 2-CXCR3) cells (Fig. S6). As a whole, we show that specific $\mathrm{T}$ cell and monocyte subsets are altered in the periphery of COVID-19 patients, highlighting systemic immune responses to the disease. 


\section{DISCUSSION}

In our current study, we examined myeloid and $\mathrm{T}$ cell heterogeneity in subjects recovering from mild, moderate, and severe COVID-19. Specifically, we identified 10 clusters of monocytes/DCs and 17 clusters of $\mathrm{T}$ lymphocytes via mass cytometry. Of these, clusters of intermediate and nonclassical monocytes, as well as $\mathrm{CD}^{+} \mathrm{T}_{\mathrm{SCM}}$ cells, correlated with disease severity, coagulation factors, and inflammatory indices. We also found that intermediate monocytes tightly correlated with loss of $\mathrm{T}_{\mathrm{N}}$ cells and an increased abundance of effector T cells, PD-1 ${ }^{+}$'exhausted' CD4 $4^{+} \mathrm{T}_{\mathrm{EM}} 2$ cells, and CD8 ${ }^{+} \mathrm{T}_{\mathrm{EM}} 2$ cells. The nMo1 cluster also correlated with $\mathrm{CD}^{+} \mathrm{T}_{\mathrm{EM}} 1$ cells and disease severity.

The mildly ill individuals in our cohort were much younger than the moderately and severely ill individuals, while the latter two groups were similar in mean age (see Methods for details). This observation mirrored published findings that aged individuals are more susceptible to severe cases of COVID-19 (66), but is confounded by the fact that the mildly ill individuals represented a specific demographic (healthcare workers). Still, many pathological states are associated with age, including cardiovascular disease and cancer. A recent study by our laboratory demonstrated that human monocyte frequencies are minimally impacted by age (27). In contrast, naive T cell subsets markedly decrease with age and this change is accompanied by a concomitant increase in memory $\mathrm{T}$ cells $(56,67,68)$. $\mathrm{CD}^{+} \mathrm{T}_{\mathrm{SCM}}$ cells embodied the sole $\mathrm{T}$ cell population found to be significantly attenuated in moderate versus severe disease. Moreover, $\mathrm{T}_{\mathrm{SCM}}$ cell frequencies were negatively correlated with the coagulation parameter INR in COVID-19 individuals. $\mathrm{T}_{\mathrm{SCM}}$ cells are antigen-experienced and the least-differentiated memory subset. In humans, $\mathrm{T}_{\mathrm{SCM}}$ cells are distinguished from $\mathrm{T}_{\mathrm{N}}$ cells by expression of CD95 (Fas or Apo-1). Increased $\mathrm{T}_{\mathrm{SCM}}$ cell frequencies are associated with improved response to viral infections. For example, $\mathrm{HIV}^{+}$ individuals on highly active antiretroviral therapy display increased $\mathrm{CD}^{+} \mathrm{T}_{\mathrm{SCM}}$ frequencies that negatively correlate with viral loads and $\mathrm{CD}^{+} \mathrm{T}$ cell counts (69). A recent study found no differences in peripheral $\mathrm{CD}^{+}$or $\mathrm{CD}^{+} \mathrm{T}_{\mathrm{SCM}}$ frequencies between COVID-19 donors and age-matched controls (26). 
However, as these data were not stratified by illness severity, longitudinal quantification of $\mathrm{T}$ cell subsets during COVID-19 is still needed.

Nonclassical monocytes play important anti-tumoral and atheroprotective roles $(48,70)$ and are distinguished by their ability to patrol the vasculature $(71,72)$. Vascular patrolling by nonclassical monocytes occurs during both steady-state and inflammation and allows surveillance against invading pathogens and maintenance of vascular endothelial homeostasis (73). We reported that the vascular endothelium is activated in mice that have nonclassical monocytes unable to patrol the vasculature due to disruptions in integrin signaling (39). We hypothesize that both nMo1 and nMo2 monocytes patrol the vasculature, but this has yet to be tested. We have found that nMo2 lowly express CXCR6, are migratory towards CXCL16, and highly phagocytic (27). We and others have found that $\mathrm{Slan}^{+} \mathrm{nMo} 2$ monocytes make IL-12 (44), recruit NK cells (48), and activate T cells $(46,47)$. We speculate that loss of nMo2 cells in moderate and severe patients could be one explanation for the coagulation issues that arise in SARSCoV-2 infection, in part due to the lack of the presence of these cells to maintain vascular endothelial homeostasis. However, studies have also shown that monocytes can release tissue factor, which is important for coagulation (74), so this could be another mechanism by which monocyte changes could impact coagulation in the vasculature of COVID-19 patients. Unfortunately, tissue factor was not included as a CyTOF marker in this study. Thus, an important next step of this study is to examine how alterations in coagulation are driven by COVID-19-based changes in monocyte subpopulations.

As we and others have long-hypothesized that all nonclassical monocytes are anti-inflammatory and anti-viral $(27,40,73,75)$, we were surprised to find that nMo1 monocytes are decreased in moderate but increased in severe COVID-19 patients. In fact, nMo1 cells appear to drive illness severity in our cohort. nMo1 cells in moderate patients displayed higher HLA-DR, PD-L1, and PD-L2 expression than those in severe patients, suggesting a possible exhaustion phenotype, or that they trigger $\mathrm{T}$ cell exhaustion. We also found positive correlations between these cells and levels of CRP, D-dimers, and INR. We were unable to perform functional studies on these cells, due to low amounts of sample 
availability. In lieu of this, we examined differences in marker expression between nMo1 and nMo2 cells. Differentially-enriched markers between these subpopulations included CD9 and CD36. CD9 on monocytes drives M2-like alternative activation pathways, including release of IL-10 (76). However, what drives the association of nMo1 with disease severity is unknown.

Recent work by the Amit laboratory (77) suggests that monocytes and macrophages can either be infected by, or phagocytize, SARS-CoV-2 particles. While monocytes minimally express the SARS-CoV2 receptor ACE-2, they express CD147 (also known as BSG), a potential receptor for the virus (78). Given the viral scavenging of nMo1 and nMo2 cells, nMo2 monocytes may eventually be killed by viral infection or by excess viral particle uptake. An alternative hypothesis is that activation of nMo1 cells by viral entry or viral particle uptake triggers responses by nMo1 monocytes in COVID-19 patients. Future studies should focus on testing these two hypotheses.

Our study utilizes PBMCs from convalescent COVID-19 patients to characterize immune cell responses to the disease. Examining monocytes and T cells in acute COVID-19, as well as performing longitudinal studies on samples from patients during illness onset and recovery, could further define immune cell interactions during COVID-19 progression. In summary, our findings detail previously unknown phenotypes of monocytes and T cells during COVID-19 and suggest interactions between subsets of these two cell types during the disease. 


\section{MATERIALS AND METHODS Patient Samples}

Ethical approval for this study was received from the Berkshire Research Ethics 20/SC/0155 and the Ethics committee of the La Jolla Institute for Immunology. Written, informed consent was obtained for all 30 subjects. Moderate $(n=13)$ and severe $(n=9)$ individuals were hospitalized in a teaching hospital in the south of England either in a general ward (moderate disease), or with treatment escalated to transfer to the intensive care unit (ICU) for patients with severe disease. SARS-CoV2 was confirmed by reverse transcriptase polymerase reaction (RT-PCR) or by detection of anti-spike protein antibodies performed in the clinical virology laboratory of the hospital. The mild cohort comprised eight health care workers who were not hospitalized, but COVID-19 diagnosis was confirmed via RT-PCR or serological evidence of SARS-CoV2 antibodies. All individuals supplied up to $80 \mathrm{~mL}$ of blood for research studies. Clinical and demographic data were collected from patient records for hospitalized patients, including ancestry, comorbidities, blood results, drug intervention, radiological involvement, thrombotic events, microbiology, and virology results.

The mean age of mild COVID-19 individuals was $39 \pm 2(26-50)$ years, moderate: $62 \pm 14(39-82)$ years; Severe: 56 \pm 10 (33-65) years (Mild vs. Moderate: $* * * p=0.0004$; Mild vs. Severe: $* p=0.0161$; Moderate vs. Severe: $p=0.4076$; One-Way ANOVA). Within the mild cohort, $50 \%$ were male, and within the moderate cohort, $77 \%$ of individuals were male. In the severe cohort, $78 \%$ were male. The mild cohort comprised 7 White/British individuals, and 1 White/Other individual. The moderate cohort comprised 2 Indian, 9 White/British, 1 Black/British, and 1 White/Other participant. The severe cohort comprised 5 White/British, 1 Black/British, 2 Indian, and 1 White/Other participant.

The average hospital stay of moderate individuals was $7.8 \pm 3.2$ days, while severe individuals were in the hospital for an average of $20 \pm 11.8$ days $(* * * p=0.004$; Mann-whitney test). Five of 9 individuals $(55.5 \%)$ within the severe cohort were intubated. Six individuals reported previous respiratory airway 
issues, including 3 within the moderate cohort reporting history of asthma or chronic obstructive pulmonary disease (COPD), 1 within the severe cohort with asthma, and two moderately affected individuals with COPD. Four of nine (44\%) individuals within the severe cohort reported hypertension, while 1 of $13(7.7 \%)$ individuals within the moderate cohort reported hypertension $(p=0.1159$; Fisher's exact test). Two individuals (2/13) within the moderate cohort reported a previous DVT/arrhythmia and myocardial infarction, respectively. Two of thirteen individuals within the moderate cohort were diagnosed with cancer ( $p=0.4935$; Fisher's exact test). Three of nine $(33.3 \%)$ individuals within the severe cohort had diabetes, in contrast to none in the moderate cohort (Moderate vs. Severe; $p=0.0545$; Fisher's exact test). All moderate and severe individuals received antibiotics during hospitalization. Two of nine severe individuals were participants of the remdesivir and REMCAP trials, respectively. All hospitalized individuals recovered and were discharged from the hospital.

\section{Sample processing}

PBMCs were thawed from the recovered COVID-19 individuals, barcoded with CD45, and stained with a monocyte-T cell panel, as previously described (27). Mild, moderate, and severe COVID-19 individuals were assayed over a period of five distinct CyTOF runs (Round 1: 3 moderate and 2 severe disease, Round 2: 5 moderate and 2 severe disease, Round 3: 2 mild, 5 moderate, and 1 severe disease, Round 4: 4 severe disease, and Round 5: 6 mild disease). To identify batch effects, a technical control of healthy PBMCs was CD45-barcoded and spiked into each individual sample. Tuning was performed every 6hrs. Data was normalized using the Matlab-based NormalizerR2013a_Win64. Live cells were gated via DNA1 by DNA-2 and were Cisplatin ${ }^{\text {lo }}$.

\section{CyTOF analysis - Debarcoding and batch correction}

Each CyTOF sample was debarcoded from the original fcs file (with spike-in healthy PBMC samples as a control) using a deconvolution algorithm (79) implemented in the CATALYST Bioconductor package. We reevaluated the markers used for barcoding by plotting the density of corresponding markers in each of fcs files after debarcoding. First, each fcs file was normalized using an arcsinh transformation 
(cofactor=5). Then, we took advantage of the same spike-in healthy control for the batch correction, using a quantile normalization method for the pooled distribution of each batch (a pair of sample and spike-in control), as implemented in the function normalizeBatch from the CYDAR Bioconductor package (80). The density plot of all protein markers was plotted before and after normalization; representative correction plots of CD3 (A), CD4 (B), CD8 (C), and CD11a (E), CD14 (F), and CD16 (G) are shown (Fig. S7).

\section{CyTOF analysis - High dimensional reduction and clustering}

Mass cytometry analysis was performed similarly to the pipeline described in our previous work (27). We used the FlowSOM clustering method (21), a self-organizing map (SOM) machine learning method, with default parameters (SOM grid 10x10 corresponds to 100 subclusters) to identify major cell-types from $\mathrm{CD}_{4} 5^{+}$live cells as well as to determine the heterogeneity of myeloid and $\mathrm{T}$ cell compartments. Major cell-types were identified using well-known lineage markers. Consensus clustering was used to justify the optimal number of clusters from $\mathrm{k}=2$ to $\mathrm{k}=30$ to evaluate the robustness of the number of clusters based on relatively decrease in area under the CDF (Cumulative Distribution Function) curve (81). The clusters were merged based on their similarity of their representative protein markers. Heatmaps of median protein expression were produced using the pheatmap R package (v0.2). UMAP deduction was done using the umap R package (v0.2.3.1), a wrapper for Python package ‘umap-learn’. For T cell clustering in Figure 3, $\mathrm{CD} 14^{\text {lo }} \mathrm{CD} 66 \mathrm{~b} / \mathrm{Siglec} / \mathrm{CD} 19^{\text {lo }} \mathrm{CD} 3^{+} \mathrm{CD} 4^{+}$and $\mathrm{CD}^{+} \mathrm{CD}^{+} \mathrm{T}$ cells were gated in FlowJo v10.3 prior to downstream FlowSOM clustering. Differential expression analysis of combined features of Tcell/myeloid subsets and surface markers (487 total features) were used to select top features differentiating disease severity (fold-change cutoff of 1), as shown in Figure S6.

\section{Statistical analysis}

Differences in immune subpopulation frequencies were evaluated using Wilcox rank-sum tests. Correlation of $\mathrm{T}$ cell and monocyte/DC subset frequencies with one other, as well as with clinical parameters, was performed using Spearman rank correlation tests. 


\section{REFERENCES AND NOTES}

1. W. J. Guan et al., Clinical Characteristics of Coronavirus Disease 2019 in China. N Engl J Med 382, 1708-1720 (2020).

2. S. Cui, S. Chen, X. Li, S. Liu, F. Wang, Prevalence of venous thromboembolism in patients with severe novel coronavirus pneumonia. J Thromb Haemost, (2020).

3. J. Helms et al., High risk of thrombosis in patients with severe SARS-CoV-2 infection: a multicenter prospective cohort study. Intensive Care Med, (2020).

4. F. A. Klok et al., Incidence of thrombotic complications in critically ill ICU patients with COVID19. Thromb Res, (2020).

5. W. Tian et al., Predictors of mortality in hospitalized COVID-19 patients: A systematic review and meta-analysis. J Med Virol, (2020).

6. D. Wang et al., Clinical Characteristics of 138 Hospitalized Patients With 2019 Novel Coronavirus-Infected Pneumonia in Wuhan, China. JAMA, (2020).

7. J. Yang et al., Prevalence of comorbidities and its effects in coronavirus disease 2019 patients: A systematic review and meta-analysis. Int J Infect Dis 94, 91-95 (2020).

8. Z. Zheng et al., Risk factors of critical \& mortal COVID-19 cases: A systematic literature review and meta-analysis. J Infect, (2020).

9. F. Zhou et al., Clinical course and risk factors for mortality of adult inpatients with COVID-19 in Wuhan, China: a retrospective cohort study. Lancet 395, 1054-1062 (2020).

10. K. J. Clerkin et al., Coronavirus Disease 2019 (COVID-19) and Cardiovascular Disease. Circulation, (2020).

11. E. J. Giamarellos-Bourboulis et al., Complex Immune Dysregulation in COVID-19 Patients with Severe Respiratory Failure. Cell Host Microbe 27, 992-1000 e1003 (2020).

12. E. Z. Ong et al., A Dynamic Immune Response Shapes COVID-19 Progression. Cell Host Microbe 27, 879-882 e872 (2020).

13. M. Merad, J. C. Martin, Pathological inflammation in patients with COVID-19: a key role for monocytes and macrophages. Nat Rev Immunol 20, 355-362 (2020).

14. J. Schulte-Schrepping et al., Suppressive myeloid cells are a hallmark of severe COVID-19. medRxiv, (2020).

15. D. Mathew et al., Deep immune profiling of COVID-19 patients reveals patient heterogeneity and distinct immunotypes with implications for therapeutic interventions. bioRxiv, (2020).

16. G. Chen et al., Clinical and immunological features of severe and moderate coronavirus disease 2019. J Clin Invest 130, 2620-2629 (2020).

17. N. Vabret et al., Immunology of COVID-19: Current State of the Science. Immunity 52, 910-941 (2020).

18. A. J. Wilk et al., A single-cell atlas of the peripheral immune response in patients with severe COVID-19. Nat Med, (2020).

19. H. Y. Zheng et al., Elevated exhaustion levels and reduced functional diversity of T cells in peripheral blood may predict severe progression in COVID-19 patients. Cell Mol Immunol 17, 541-543 (2020).

20. M. Zheng et al., Functional exhaustion of antiviral lymphocytes in COVID-19 patients. Cell Mol Immunol 17, 533-535 (2020). 
21. S. Van Gassen et al., FlowSOM: Using self-organizing maps for visualization and interpretation of cytometry data. Cytometry A 87, 636-645 (2015).

22. E. Becht et al., Dimensionality reduction for visualizing single-cell data using UMAP. Nat Biotechnol, (2018).

23. Q. Zhao et al., Lymphopenia is associated with severe coronavirus disease 2019 (COVID-19) infections: A systemic review and meta-analysis. Int J Infect Dis 96, 131-135 (2020).

24. L. Tan et al., Lymphopenia predicts disease severity of COVID-19: a descriptive and predictive study. Signal Transduct Target Ther 5, 33 (2020).

25. C. Huang et al., Clinical features of patients infected with 2019 novel coronavirus in Wuhan, China. Lancet 395, 497-506 (2020).

26. S. De Biasi et al., Marked T cell activation, senescence, exhaustion and skewing towards TH17 in patients with COVID-19 pneumonia. Nat Commun 11, 3434 (2020).

27. A. A. J. Hamers et al., Human Monocyte Heterogeneity as Revealed by High-Dimensional Mass Cytometry. Arterioscler Thromb Vasc Biol 39, 25-36 (2019).

28. C. Papewalis et al., IFN-alpha skews monocytes into CD56+-expressing dendritic cells with potent functional activities in vitro and in vivo. J Immunol 180, 1462-1470 (2008).

29. G. Rothe et al., Peripheral blood mononuclear phagocyte subpopulations as cellular markers in hypercholesterolemia. Arterioscler Thromb Vasc Biol 16, 1437-1447 (1996).

30. J. S. Lee et al., Immunophenotyping of COVID-19 and influenza highlights the role of type I interferons in development of severe COVID-19. Sci Immunol 5, (2020).

31. E. M. Bradshaw et al., CD33 Alzheimer's disease locus: altered monocyte function and amyloid biology. Nat Neurosci 16, 848-850 (2013).

32. M. Wildgruber et al., The "Intermediate" CD14(++)CD16(+) monocyte subset increases in severe peripheral artery disease in humans. Sci Rep 6, 39483 (2016).

33. M. Yang et al., Proinflammatory CD14+CD16+ monocytes are associated with microinflammation in patients with type 2 diabetes mellitus and diabetic nephropathy uremia. Inflammation 35, 388-396 (2012).

34. J. W. Lee et al., Proinflammatory CD14(+)CD16(+) monocytes are associated with vascular stiffness in predialysis patients with chronic kidney disease. Kidney Res Clin Pract 32, 147-152 (2013).

35. A. Mildner et al., Genomic Characterization of Murine Monocytes Reveals C/EBPbeta Transcription Factor Dependence of Ly6C(-) Cells. Immunity 46, 849-862 e847 (2017).

36. M. Guilliams, A. Mildner, S. Yona, Developmental and Functional Heterogeneity of Monocytes. Immunity 49, 595-613 (2018).

37. A. M. Zawada et al., SuperSAGE evidence for CD14++CD16+ monocytes as a third monocyte subset. Blood 118, e50-61 (2011).

38. Y. Zhou, B. Fu, X. Zheng, D. Wang, Aberrant pathogenic GM-CSF+ T cells and inflammatory CD14+CD16+ monocytes in severe pulmonary syndrome patients of a new coronavirus. bioRxiv, (2020).

39. P. M. Marcovecchio et al., Kindlin-3 is essential for patrolling and phagocytosis functions of nonclassical monocytes during metastatic cancer surveillance. J Leukoc Biol, (2020).

40. J. Cros et al., Human CD14dim monocytes patrol and sense nucleic acids and viruses via TLR7 and TLR8 receptors. Immunity 33, 375-386 (2010).

41. W. Vermi et al., slan(+) Monocytes and Macrophages Mediate CD20-Dependent B-cell Lymphoma Elimination via ADCC and ADCP. Cancer Res 78, 3544-3559 (2018).

42. K. Thomas et al., Accumulation and therapeutic modulation of 6-sulfo LacNAc(+) dendritic cells in multiple sclerosis. Neurol Neuroimmunol Neuroinflamm 1, e33 (2014).

43. T. P. Hofer, A. A. van de Loosdrecht, C. Stahl-Hennig, M. A. Cassatella, L. Ziegler-Heitbrock, 6Sulfo LacNAc (Slan) as a Marker for Non-classical Monocytes. Front Immunol 10, 2052 (2019). 
44. K. Schakel et al., Human 6-sulfo LacNAc-expressing dendritic cells are principal producers of early interleukin-12 and are controlled by erythrocytes. Immunity 24, 767-777 (2006).

45. F. Ahmad, T. Dobel, M. Schmitz, K. Schakel, Current Concepts on 6-sulfo LacNAc Expressing Monocytes (slanMo). Front Immunol 10, 948 (2019).

46. K. Schakel et al., 6-Sulfo LacNAc, a novel carbohydrate modification of PSGL-1, defines an inflammatory type of human dendritic cells. Immunity 17, 289-301 (2002).

47. K. Schakel et al., A novel dendritic cell population in human blood: one-step immunomagnetic isolation by a specific $\mathrm{mAb}$ (M-DC8) and in vitro priming of cytotoxic T lymphocytes. Eur $J$ Immunol 28, 4084-4093 (1998).

48. R. N. Hanna et al., Patrolling monocytes control tumor metastasis to the lung. Science 350, 985 990 (2015).

49. M. Schmitz et al., Tumoricidal potential of native blood dendritic cells: direct tumor cell killing and activation of NK cell-mediated cytotoxicity. J Immunol 174, 4127-4134 (2005).

50. A. A. Schonherz et al., Normal myeloid progenitor cell subset-associated gene signatures for acute myeloid leukaemia subtyping with prognostic impact. PLoS One 15, e0229593 (2020).

51. F. Sallusto, D. Lenig, C. R. Mackay, A. Lanzavecchia, Flexible programs of chemokine receptor expression on human polarized T helper 1 and 2 lymphocytes. $J$ Exp Med 187, 875-883 (1998).

52. A. Langenkamp et al., Kinetics and expression patterns of chemokine receptors in human CD4+ T lymphocytes primed by myeloid or plasmacytoid dendritic cells. Eur J Immunol 33, 474-482 (2003).

53. G. De Simone et al., CXCR3 Identifies Human Naive CD8(+) T Cells with Enhanced Effector Differentiation Potential. J Immunol 203, 3179-3189 (2019).

54. J. Z. Long et al., Metabolomics annotates ABHD3 as a physiologic regulator of medium-chain phospholipids. Nat Chem Biol 7, 763-765 (2011).

55. E. Lugli et al., Identification, isolation and in vitro expansion of human and nonhuman primate $\mathrm{T}$ stem cell memory cells. Nat Protoc 8, 33-42 (2013).

56. F. F. Fagnoni et al., Shortage of circulating naive CD8(+) T cells provides new insights on immunodeficiency in aging. Blood 95, 2860-2868 (2000).

57. M. Pinti et al., Aging of the immune system: Focus on inflammation and vaccination. Eur $J$ Immunol 46, 2286-2301 (2016).

58. L. Gattinoni et al., A human memory T cell subset with stem cell-like properties. Nat Med 17, 1290-1297 (2011).

59. N. Cieri et al., IL-7 and IL-15 instruct the generation of human memory stem T cells from naive precursors. Blood 121, 573-584 (2013).

60. D. Weiskopf et al., Phenotype and kinetics of SARS-CoV-2-specific T cells in COVID-19 patients with acute respiratory distress syndrome. Sci Immunol 5, (2020).

61. M. T. Zilber et al., MHC class II/CD38/CD9: a lipid-raft-dependent signaling complex in human monocytes. Blood 106, 3074-3081 (2005).

62. P. A. Beavis, J. Stagg, P. K. Darcy, M. J. Smyth, CD73: a potent suppressor of antitumor immune responses. Trends Immunol 33, 231-237 (2012).

63. J. J. Kobie et al., T regulatory and primed uncommitted CD4 T cells express CD73, which suppresses effector CD4 T cells by converting 5'-adenosine monophosphate to adenosine. $J$ Immunol 177, 6780-6786 (2006).

64. C. Qin et al., Dysregulation of immune response in patients with COVID-19 in Wuhan, China. Clin Infect Dis, (2020).

65. B. J. Meckiff et al., Single-cell transcriptomic analysis of SARS-CoV-2 reactive CD4+ T cells. bioRxiv, (2020).

66. W. C. Koff, M. A. Williams, Covid-19 and Immunity in Aging Populations - A New Research Agenda. N Engl J Med, (2020). 
67. C. Franceschi et al., Inflamm-aging. An evolutionary perspective on immunosenescence. Annals of the New York Academy of Sciences 908, 244-254 (2000).

68. S. Salvioli et al., Immune system, cell senescence, aging and longevity--inflamm-aging reappraised. Curr Pharm Des 19, 1675-1679 (2013).

69. S. P. Ribeiro et al., The CD8(+) memory stem T cell (T(SCM)) subset is associated with improved prognosis in chronic HIV-1 infection. J Virol 88, 13836-13844 (2014).

70. R. N. Hanna et al., NR4A1 (Nur77) deletion polarizes macrophages toward an inflammatory phenotype and increases atherosclerosis. Circ Res 110, 416-427 (2012).

71. J. L. Collison, L. M. Carlin, M. Eichmann, F. Geissmann, M. Peakman, Heterogeneity in the Locomotory Behavior of Human Monocyte Subsets over Human Vascular Endothelium In Vitro. $J$ Immunol 195, 1162-1170 (2015).

72. C. Auffray et al., Monitoring of blood vessels and tissues by a population of monocytes with patrolling behavior. Science 317, 666-670 (2007).

73. L. M. Carlin et al., Nr4a1-dependent Ly6C(low) monocytes monitor endothelial cells and orchestrate their disposal. Cell 153, 362-375 (2013).

74. Y. Hisada, N. Mackman, Measurement of tissue factor activity in extracellular vesicles from human plasma samples. Res Pract Thromb Haemost 3, 44-48 (2019).

75. M. A. Ingersoll et al., Comparison of gene expression profiles between human and mouse monocyte subsets. Blood 115, e10-19 (2010).

76. C. Brosseau, L. Colas, A. Magnan, S. Brouard, CD9 Tetraspanin: A New Pathway for the Regulation of Inflammation? Front Immunol 9, 2316 (2018).

77. P. G. Bost, A.; Liu, Y.; Bendjelal, Y.; Xu, G.; David, E.; Blecher-Gonen.; Cohen, M.; Medaglia, C.; Li, H.; Deczkowska, A.; Zhang, S.; Schwikowski, B.; Zhang, Z.; Amit, I., Host-viral infection maps reveal signatures of severe COVID-19 patients. Cell Epub ahead of print., (2020).

78. K. Wang et al., SARS-CoV-2 invades host cells via a novel route: CD147- spike protein. bioRxiv, (2020).

79. E. R. Zunder et al., Palladium-based mass tag cell barcoding with a doublet-filtering scheme and single-cell deconvolution algorithm. Nat Protoc 10, 316-333 (2015).

80. A. T. L. Lun, A. C. Richard, J. C. Marioni, Testing for differential abundance in mass cytometry data. Nat Methods 14, 707-709 (2017).

81. M. D. Wilkerson, D. N. Hayes, ConsensusClusterPlus: a class discovery tool with confidence assessments and item tracking. Bioinformatics 26, 1572-1573 (2010). 


\section{ACKNOWLEDGEMENTS}

We would like to thank Cheryl Kim and the LJI Flow Cytometry Core for assistance. We also thank Luke Smith for patient recruitment and sample collection, Callum Dixon, Benjamin Johnson, Lydia Scarlett and Silvia Austin for collection of clinical data, and Céline Galloway, Oliver Wood, Katy McCann and Lindsey Chudley for sample processing. Funding: The Fluidigm CyTOF Helios mass cytometer was supported by 1S10OD018499 (to Shane Crotty, LJI). Dr. Padgett has been supported by T32 AI125279, 19POST34450020, and F32 HL146069-01A1 (to L.E.P.). This work was supported in part by the Wessex Clinical Research Network and National Institute for Health Research UK. Author Contributions: Dr. Padgett designed and performed the experiments, generated figures, and wrote the manuscript. Dr. Dinh analyzed all CyTOF data and performed correlational analyses. Dr. Chee recruited and cared for patients and obtained blood samples. Dr. Olingy assisted with experimental design. Ms. Wu assisted with antibody conjugations and CyTOF analysis. Dr. Araujo participated in study design, contributed to writing and editing the manuscript, and participated in helpful discussions. Dr. Vijayanand participated in helpful discussions. Dr. Ottensmeier led the clinical study in the UK, edited the manuscript, and participated in helpful discussions. Dr. Hedrick directed the study and wrote the manuscript. Competing interests: The authors declare that they have no competing interests in relation to this work. Data and Materials Availability: Complete CyTOF panels used including metals and antibody clones are listed. All CyTOF data will be available at http://flowrepository.org/. 
A. Recovered COVID-19 Individuals
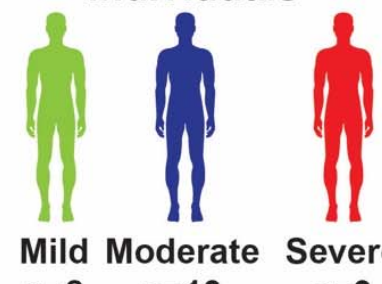

$n=8 \quad n=13$

B.

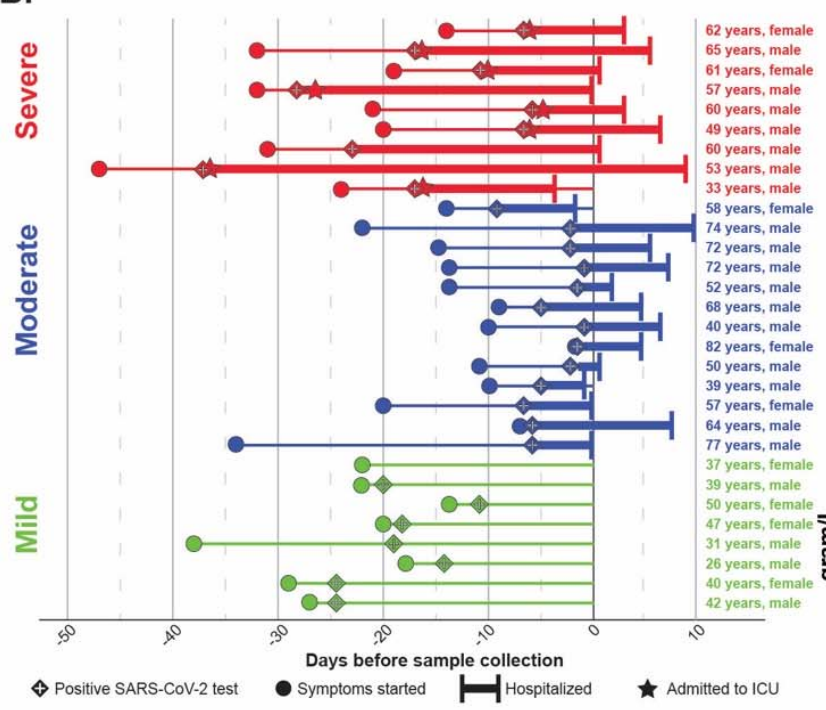

PBMCs

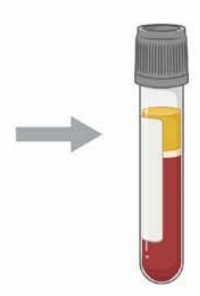

Monocyte and $\mathrm{T}$ cell immunoprofiling by CyTOF

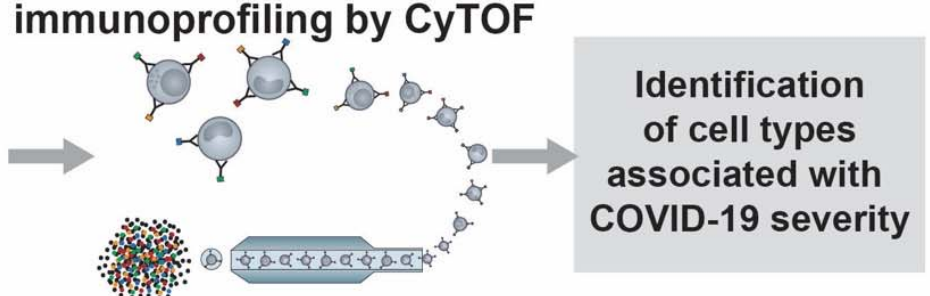

C.

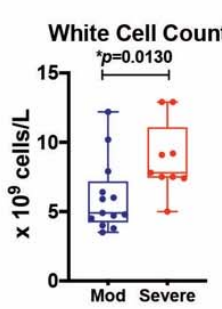

G.

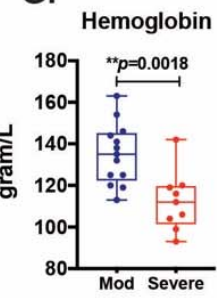

D.

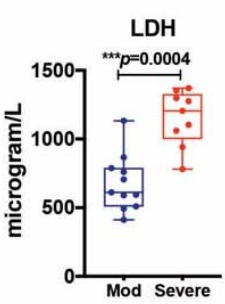

H.

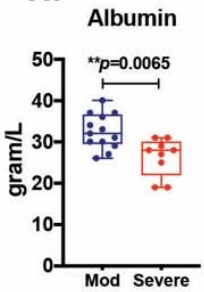

E.

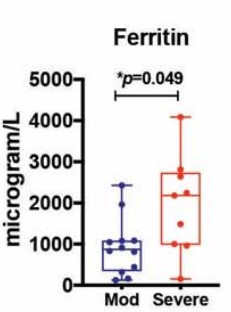

F.

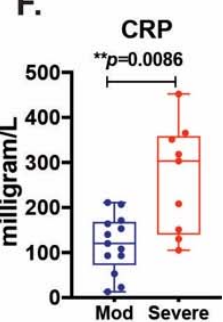

I.

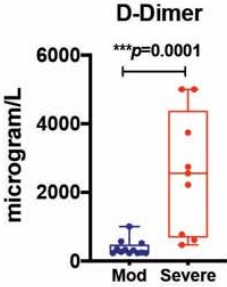

J.

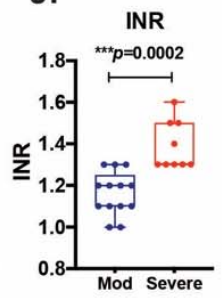

K.
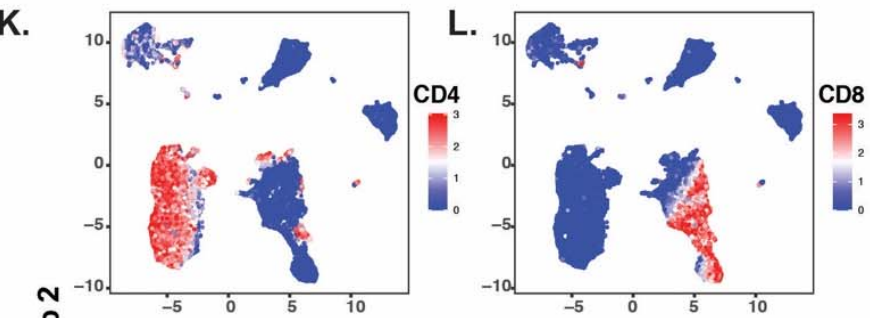

M.
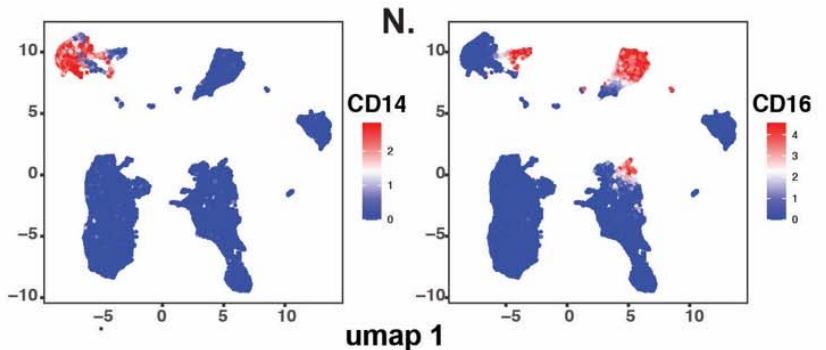

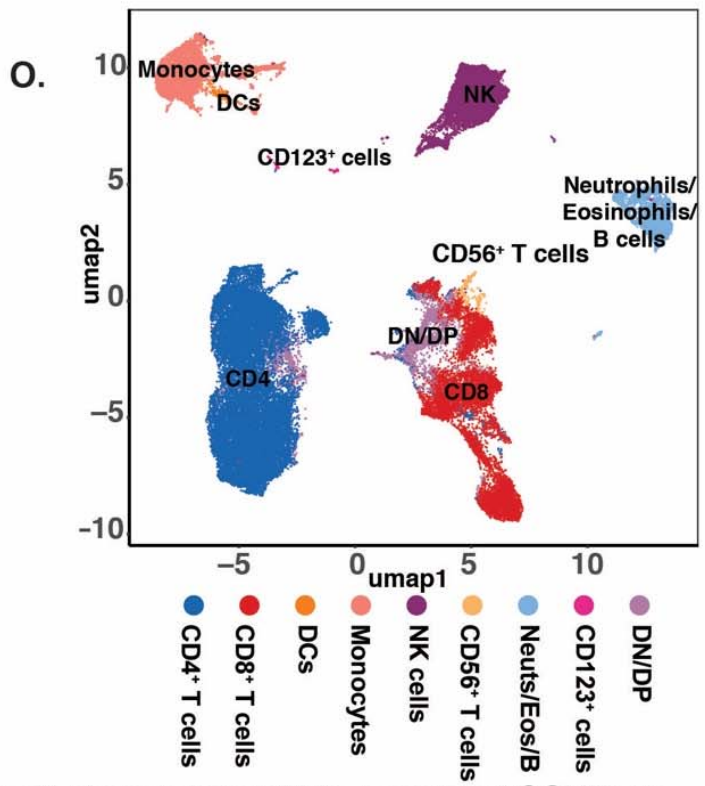

Figure 1. Experimental schematic for profiling monocyte and T cell phenotypes within recovered COVID-19 individuals. Monocyte and T cell immunoprofiling was performed via mass cytometry within PBMCs from mild $(n=8)$, moderate $(n=13)$, and severe $(n=9)$ individuals $(A)$. Patient cohort overview, depicting age, sex, COVID-19 severity, hospitalization, with all individuals aligned to the time of sample collection (B). Box and whisker plots of white blood cell counts $(\mathbf{C})$, lactate dehydrogenase (LDH, D), Ferritin (E), C-Reactive Protein (CRP, F), hemoglobin $(\mathbf{G})$, albumin $(\mathbf{H})$, D-dimer (I), and international normalized ratio (INR, J) within moderate $(n=13)$ and severe $(n=8)$ individuals. CD45 leukocytes were clustered and projected onto a UMAP, and expression of CD4 (K), CD8 (L), CD14 (M), and CD16 (N) were examined, leading to 9 immune populations (0). Statistical siginificance was calculated using Mann-whitney test. 
bioRxiv preprint doi: https://doi.org/10.1101/2020.07.17.209304; this version posted July 18, 2020. The copyright holder for this preprint (which was not certified by peer review) is the author/funder, who has granted bioRxiv a license to display the preprint in perpetuity. It is made available under aCC-BY-NC-ND 4.0 International license.
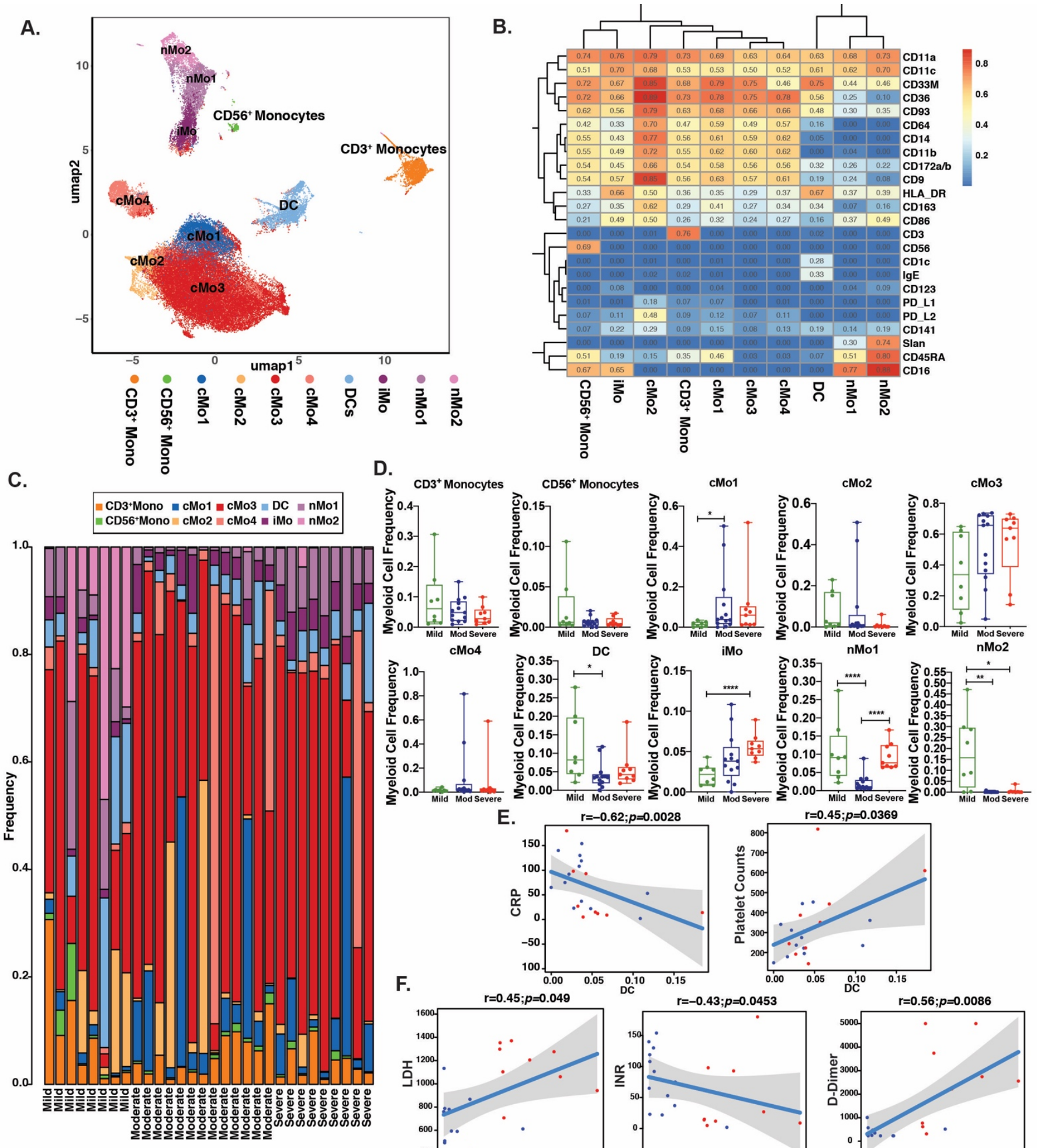

E.

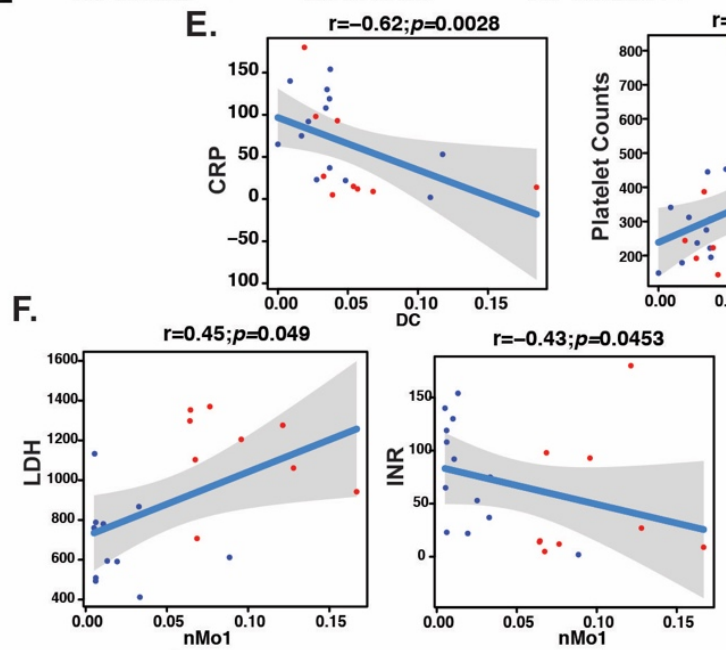
$r=0.45 ; p=0.0369$

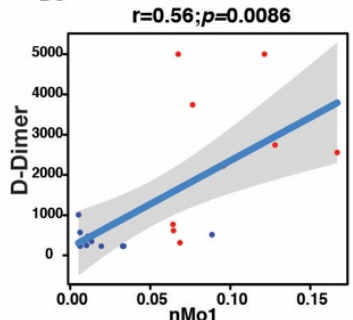

Figure 2. Monocyte immunoprofiing within recovered COVID-19 individuals. Myeloid cells were clustered and projected onto a UMAP, revealing 10 myeloid subsets (A). Heatmap of the median intensity of 24 markers across the 10 myeloid subsets (B). Stacked barplots of myeloid subset frequencies for each individual (C). Plotted frequencies of $\mathrm{CD}^{+}$monocytes, CD56 ${ }^{+}$monocytes, cMo1, cMo2, cMo3, cMo4, DC, iMo, nMo1, and nMo2 (D) for mild $(n=9)$, moderate $(n=13)$, and severe $(n=9)$ individuals. Spearman correlation plots of DCs with CRP and Platelet counts for moderate (blue) and severe (red) individuals (E). Spearman correlation plots of nMo1 with LDH, INR, and D-Dimer for moderate (blue) and severe (red) individuals (F). Statistical significance was determined using wilcoxon rank sum test. ${ }^{* \star * *} p<0.001,{ }^{* *} p<0.01,{ }^{*} p<0.05$. 
bioRxiv preprint doi: https://doi.org/10.1101/2020.07.17.209304; this version posted July 18, 2020. The copyright holder for this preprint (which

was not certified by peer review) is the author/funder, who has granted bioRxiv a license to display the preprint in perpetuity. It is made available under aCC-BY-NC-ND 4.0 International license.

A.

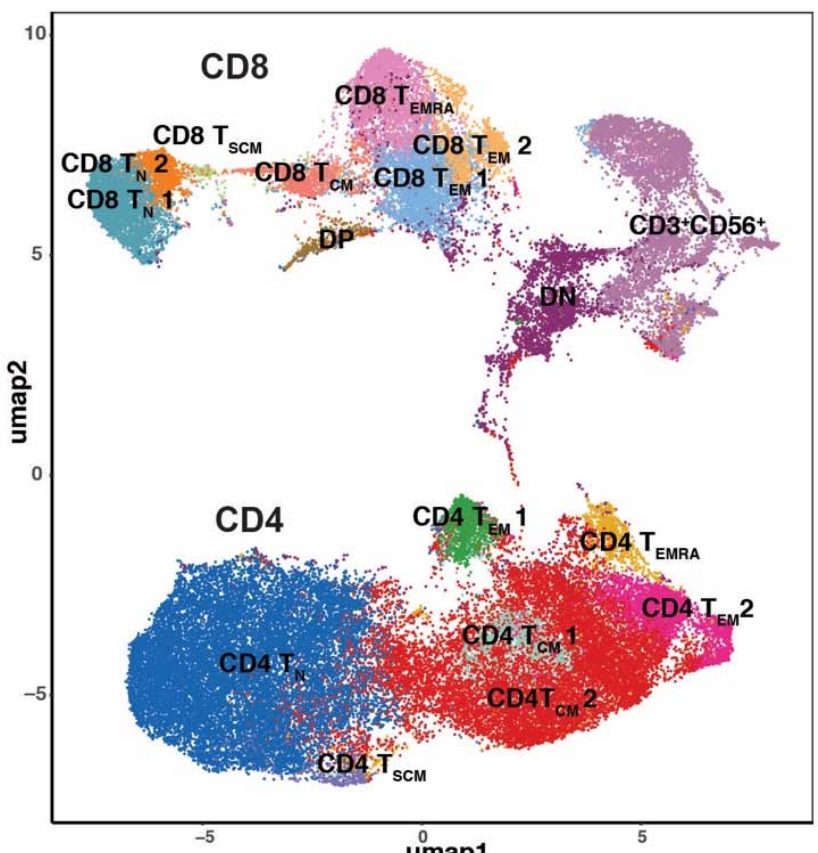

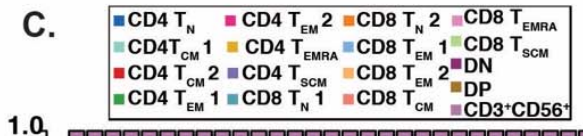
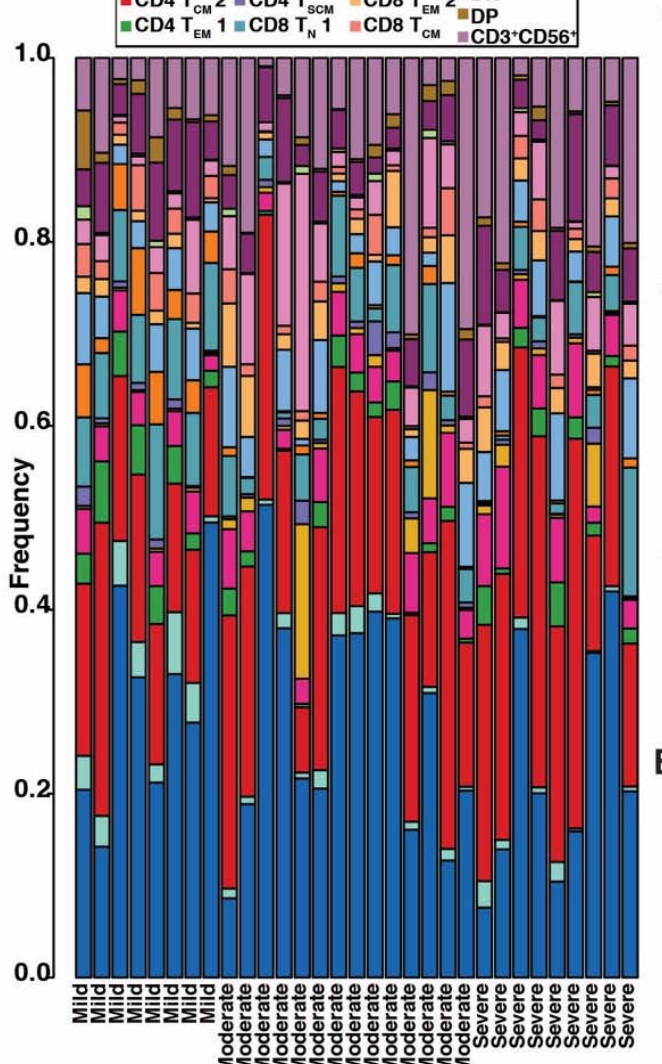

D.
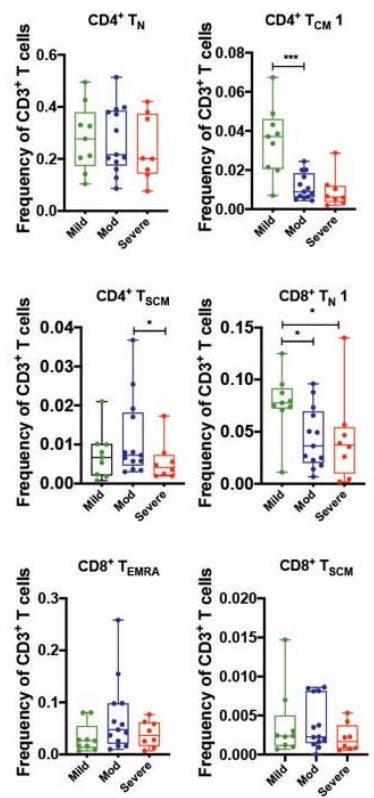

E.

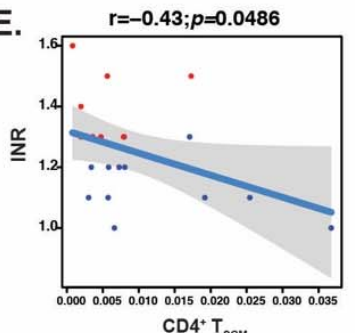

B.

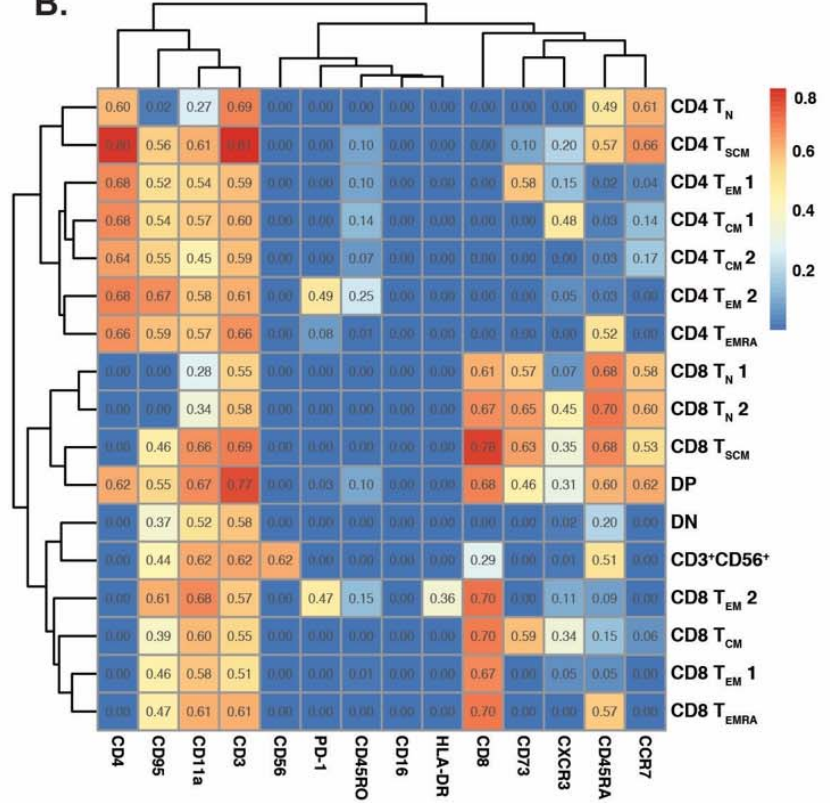

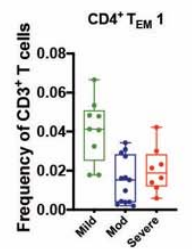
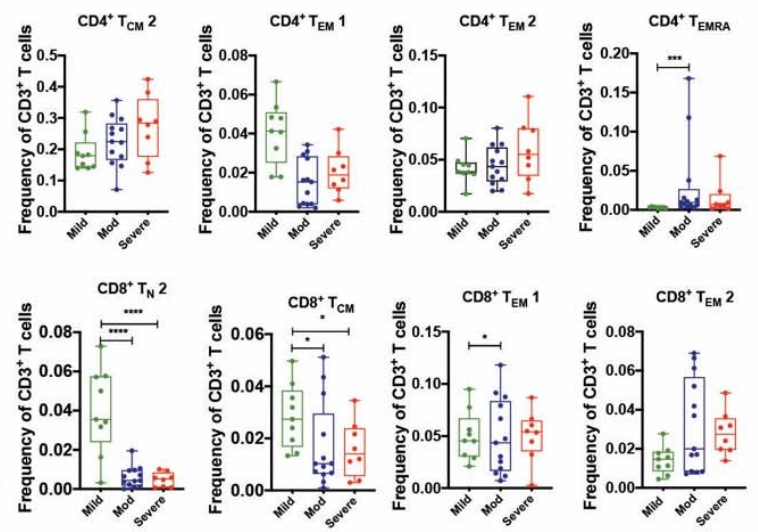
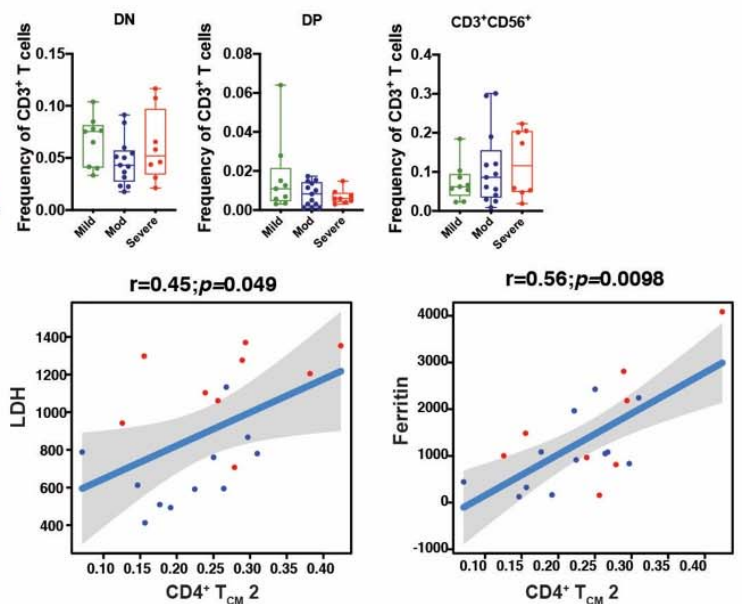

Figure 3. T cell immunoprofiing within recovered COVID-19 individuals. CD4 ${ }^{+}$and $\mathrm{CD}^{+} \mathrm{T}$ cells were clustered and projected onto a UMAP, revealing $17 \mathrm{~T}$ cell subsets (A). Heatmap of the median intensity of 24 markers across the 17 subsets (B). Stacked barplots of T cell frequencies across mild, moderate, and severe individuals (C). Plotted frequencies of the 17 T cell subsets $(D)$ for mild $(n=8)$, moderate $(n=13)$, and severe $(n=9)$ individuals. Spearman correlation plots of CD4 ${ }^{+}$ $\mathrm{T}_{\mathrm{SCM}}$ cells with INR and $\mathrm{CD} 4^{+} \mathrm{T}_{\mathrm{CM}} 2$ cells with $\mathrm{LDH}$ and Ferritin (E). Statistical significance was determined using wilcoxon rank sum test. ${ }^{* * *} p<0.001,{ }^{* *} p<0.01,{ }^{*} p<0.05$. 
A.

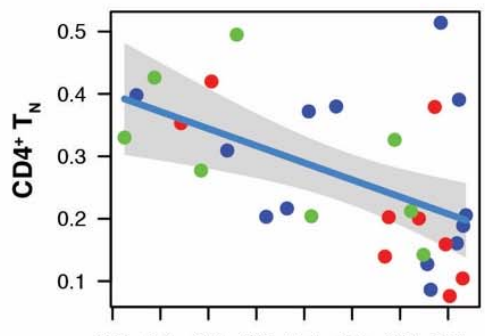

$\begin{array}{llllllll}0.0 & 0.1 & 0.2 & 0.3 & 0.4 & 0.5 & 0.6 & 0.7\end{array}$

cMo3

$r=0.63 ; p=3 e-04$

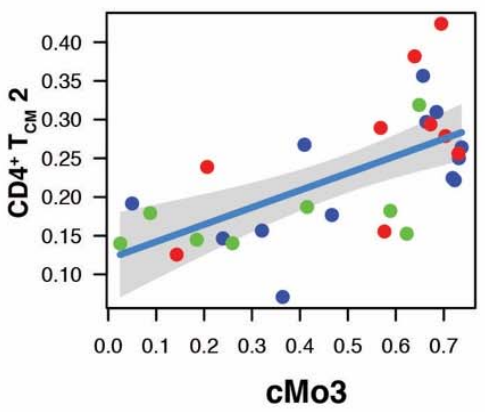

B.

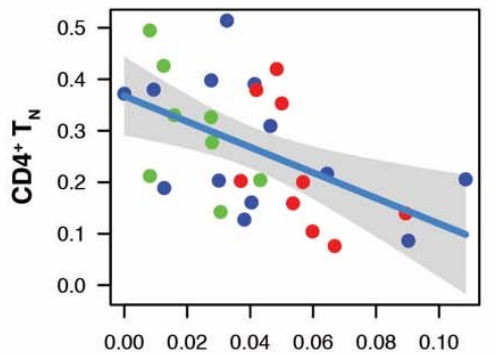

iMo

$r=0.54 ; p=0.0026$

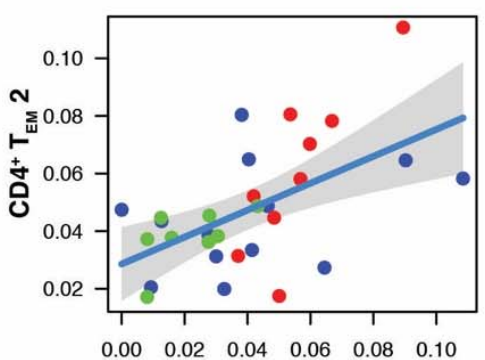

iMo

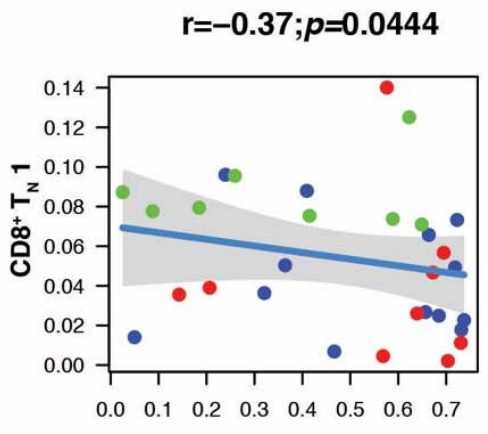

cMo3

$r=0.41 ; p=0.0268$

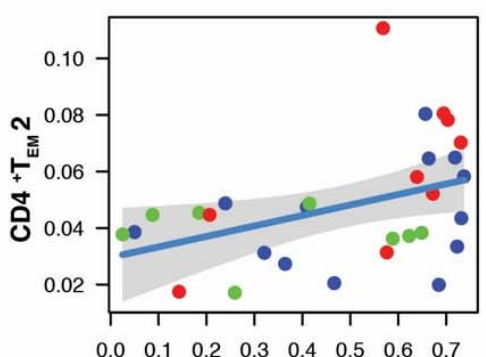

cMo3

$r=-0.42 ; p=0.0204$

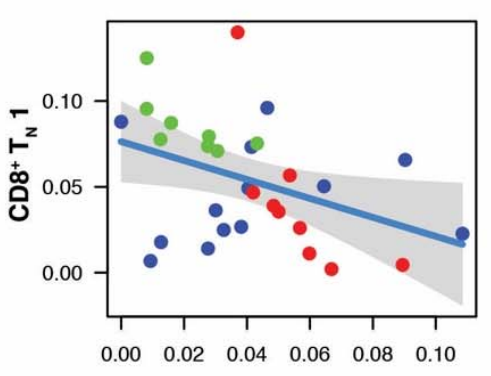

iMo

$r=0.58 ; p=0.001$

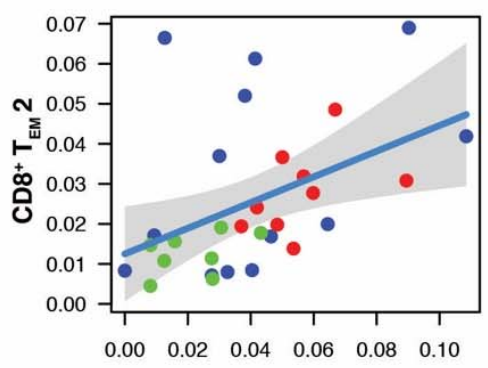

iMo $r=-0.37 ; p=0.0463$

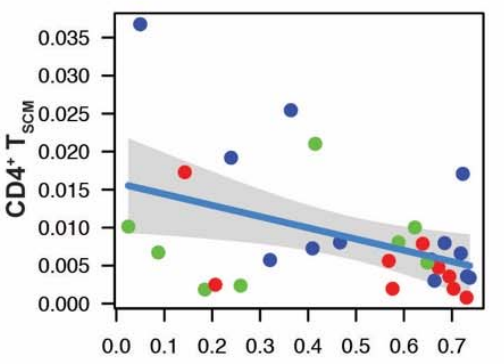

cMo3

$r=0.46 ; p=0.011$

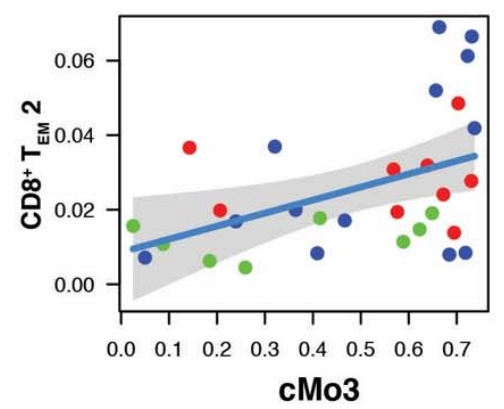

$r=0.4 ; p=0.0279$

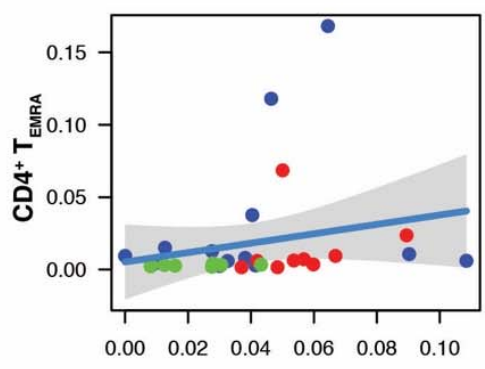

iMo

C. $\quad r=0.37 ; p=0.048$

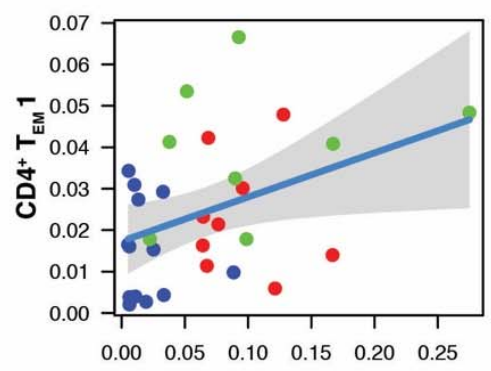

nMo1

Figure 4. Monocyte and T cell subsets are correlated in COVID-19 individuals. Spearman correlation plots of cMo3 with $\mathrm{CD}^{+} \mathrm{T}_{\mathrm{N}}, \mathrm{CD}^{+} \mathrm{T}_{\mathrm{N}} 1, \mathrm{CD}^{+} \mathrm{T}_{\mathrm{CM}} 2, \mathrm{CD}^{+} \mathrm{T}_{\mathrm{EM}} 2, \mathrm{CD}^{+} \mathrm{T}_{\mathrm{EM}} 2$ and CD4 ${ }^{+} \mathrm{T}_{\mathrm{SCM}}(\mathbf{A})$, iMo with CD4 ${ }^{+} \mathrm{T}_{\mathrm{N}}, \mathrm{CD}^{+} \mathrm{T}_{\mathrm{N}} 1, \mathrm{CD}^{+} \mathrm{T}_{\mathrm{EMRA}}$, $\mathrm{CD}^{+} \mathrm{T}_{\mathrm{EM}}$ 2, and $\mathrm{CD}^{+} \mathrm{T}_{\mathrm{EM}} 2(\mathrm{~B})$, and $\mathrm{nMO} 1$ with $\mathrm{CD}^{+} \mathrm{T}_{\mathrm{EM}} 1(\mathbf{C})$ within mild $(\mathrm{n}=8$, light green), moderate $(\mathrm{n}=13$, blue), and severe $(n=9$, red) individuals. 